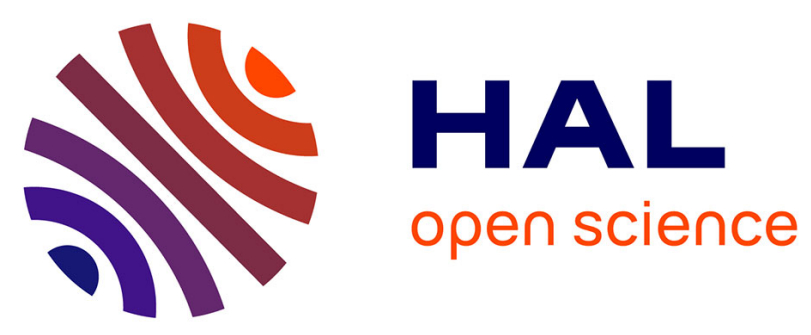

\title{
Performance Analysis and Stochastic Stability of Congestion Control Protocols
}

Eitan Altman, Konstantin Avrachenkov, Arzad A. Kherani, Balakrishna Prabhu

\section{- To cite this version:}

Eitan Altman, Konstantin Avrachenkov, Arzad A. Kherani, Balakrishna Prabhu. Performance Analysis and Stochastic Stability of Congestion Control Protocols. [Research Report] RR-5262, Inria Sophia Antipolis; INRIA. 2004, pp.56. inria-00070736

\section{HAL Id: inria-00070736 https://hal.inria.fr/inria-00070736}

Submitted on 19 May 2006

HAL is a multi-disciplinary open access archive for the deposit and dissemination of scientific research documents, whether they are published or not. The documents may come from teaching and research institutions in France or abroad, or from public or private research centers.
L'archive ouverte pluridisciplinaire HAL, est destinée au dépôt et à la diffusion de documents scientifiques de niveau recherche, publiés ou non, émanant des établissements d'enseignement et de recherche français ou étrangers, des laboratoires publics ou privés. 
INSTITUT NATIONAL DE RECHERCHE EN INFORMATIQUE ET EN AUTOMATIQUE

\title{
Performance Analysis and Stochastic Stability of Congestion Control Protocols
}

\author{
E. Altman - K. E. Avrachenkov - A. A. Kherani - B. J. Prabhu
}

$\mathbf{N}^{\circ} \mathbf{5 2 6 2}$

July 2004

Thème COM 



\title{
Performance Analysis and Stochastic Stability of Congestion Control Protocols
}

\author{
E. Altman* , K. E. Avrachenkov, A. A. Kherani ${ }^{\dagger}$, B. J. Prabhu \\ Thème COM — Systèmes communicants \\ Projets Maestro
}

Rapport de recherche $\mathrm{n}^{\circ} 5262$ - July $2004-56$ pages

\begin{abstract}
We study an Adaptive Window Protocol (AWP) with a general increase and decrease profiles in the presence of window dependent random losses. We derive a steady-state Kolmogorov equation and then obtain its solution in analytic form. We then derive from the model some monotonicity properties of the window size process. These monotonicity properties are then used to obtain a necessary and sufficient condition for stability of the window evolution process. Finally, we apply the general results to particular TCP versions such as New Reno TCP, Scalable TCP and HighSpeed TCP.
\end{abstract}

Key-words: Stochastic stability, Kolmogorov equations, Scalable TCP, HighSpeed TCP

This work was supported by grant from the Centre Franco-Indien pour la Promotion de la Recherche Avancee (CEFIPRA) under project no. 2900-IT-1.

* The work of this author was partially supported by the European Network of Excellence EURO NGI.

$\dagger$ corresponding author. 


\section{Analyse des Performances et Stabilite Stochastique des Protocoles de Contrôle de Congestion}

Résumé : Nous étudions l'Adaptive Window Protocol (AWP) avec des profils généraux d'augmentation et de diminution de la fenêtre de congestion. Nous considérons un processus de pertes dépendant de la taille de la fenêtre. Nous obtenons les équations de Kolmogorov pour l'état stationaire. Ces équations de Kolmogorov peuvent être résolues de façon analytique. Plusieurs bornes stochastiques sont établies pour le processus d'évolution de la fenêtre. Ces bornes stochastiques sont alors utilisées pour établir les conditions de stabilité du protocole AWP. Finalement, les résultats généraux sont appliqués à l'analyse de versions particulières de TCP comme New Reno TCP, Scalable TCP et HighSpeed TCP.

Mots-clés : Stabilite stochastique, Equations de Kolmogorov, Scalable TCP, HighSpeed TCP 


\section{Introduction}

Most of the performance studies of Adaptive Window Protocols (AWP) consider specific instances of the problem (for example [5, 4, 15] study Additive Increase Multiplicative Decrease (AIMD) protocols). However, various modifications to TCP are frequently proposed to address specific problems arising in various types of networks; recent examples include HighSpeed TCP [14] and Scalable TCP [8] proposed for very high bandwidth-delay product networks. These new proposals can also be viewed in the framework of Additive Increase protocols so that now the additive increase in a round-trip time is function of the current window size (it is constant in the case of standard TCP). Performance related analysis of any such protocol has always been an important issue. It is thus desirable to have a general framework (and its solution) for performance analysis of an AWP.

Since the loss process seen by a TCP sender may have origin in deliberate marking/dropping owing to some active queue management (AQM) scheme employed in the network, or could be owing to congestion losses or link errors, in general the rate of receiving such signal will depend on the window process itself (see [15] for related discussion). Hence in our study we consider a general state dependent loss rate.

It is clear that the stability of window process following a general AWP will depend on the rate at which it receives loss signals. For example, an aggressive protocol may result in very high windows for moderate loss rates and vice versa. Stability of the window process is thus interesting to study. We address the problem of finding conditions for stability of a general AWP controlled window evolution under a general state-dependent loss rate. The contribution (and organization) of this work are:

Section 2: We give a characterization of a general AWP and identify the various quantities that determine the performance of such protocols. The window evolution under a general AWP is mapped to that under an AWP with a linear increase profile (like in standard TCP). Kolmogorov equations satisfied by the stationary probability measure is then derived. Section 3: Gives conditions under which two AWPs have related stationary distribution. Furthermore, we demonstrate that the window process under multiplicative decrease protocol is also related to the workload process in a queueing system. Section 4: We give a general methodology for analysis of any such protocol while allowing for a general window dependent drop rate. The stationary distribution of a general AWP with a general loss rate is related to that of an AWP with linear increase profile and a constant loss rate. This observation is important as the latter system is easier to analyse. An analytic expression for the stationary distribution of the system with an AWP with linear increase profile and a constant loss rate is provided. Section 5 and 6: We apply the results of Section 4 to study the performance of recently proposed TCP modifications (Scalable TCP [8] and HighSpeed TCP [14]). We also refine existing result on standard AIMD protocol of TCP. We obtain some results of independent interest of queueing systems theory by relating the window process under a multiplicative increase multiplicative decrease (MIMD) protocol to the workload process in an $\mathrm{M} / \mathrm{D} / 1$ queue. This provides us with a closed form expression for the workload process in an $\mathrm{M} / \mathrm{D} / 1$ queue with bounded workload process. We also obtain a duality relation between the customer averages in a $\mathrm{D} / \mathrm{M} / 1$ queue and time average in

$\mathrm{RR} \mathrm{n}^{\circ} 5262$ 
an $\mathrm{M} / \mathrm{D} / 1$ queue (both queues with bounded workload capacity). Section 8: We obtain some stochastic ordering relations for a protocol with different bounds on window. A closed form necessary and sufficient stability condition using the stochastic ordering for the window process is established.

The proofs of all the results of this paper can be found in [32]. The report [32] also contains some additional results. Since the paper addresses many issues, for sake of making clear the context of discussion, we decided to spread the discussion on related literature across the paper instead of mentioning them all together. An extensive literature survey on TCP modeling can be found in [15].

\section{The Model}

We consider an AWP controlled persistent file transfer over an Internet (bottleneck) link. For applications using HighSpeed and Scalable TCP, this link will typically be a very high bandwidth-delay product link. We assume that the connection is long enough to see a stationary regime and that its throughput performance is governed by the steady state regime (see [5] for justification of this assumption). Required conditions for existence of such regime are detailed in a later section. Recent applications using HighSpeed TCP and Scalable TCP typically transfer very large volume files and hence studying persistent transfers is justified in such cases. We model the process of losses as a Poisson process with a time varying intensity that depends on the instantaneous window size of the AWP [15]. These losses could be owing to congestion losses, random link losses or to some deliberate packet marking/dropping by the router buffer using an AQM. As is common in related studies $([1,4,5])$, we consider the evolution of window as an infinitely divisible fluid. Details of the model are given below.

Let $x_{t}$ denote the window size of the AWP at time instant $t$ (note that we are not specifying the initial window size $x_{0}$ here, thus assuming a stationary window process). We now give the description of the window evolution. In case of no loss, the window increase in time interval $[t, t+\Delta]$ is given by,

$$
x_{t+\Delta}=x_{t}+f\left(x_{t}\right) \Delta+o(\Delta),
$$

where $f(\cdot)$ is a Lipschitz continuous function bounded below by some positive quantity. We also assume that there is a lower bound on the window size, denoted $x_{\min }$.

The increase in window can not continue for ever because drops owing to congestion or channel losses or AQM marking can occur at random instants in time ${ }^{1}$. Let $N(t)$ be the counting process corresponding to the loss events, i.e., $N(t)-N(t-u)$ is the number of losses in time interval $(t-u, t]$. In what follows we assume that $N(t)$ is a Poisson process with time varying intensity. Further, we assume that the instantaneous rate of the $N(t)$

\footnotetext{
${ }^{1}$ Congestion losses occur also when the window size reaches the practical limit of the total round trip pipe size (sum of the link bandwidth-delay product and the router buffer). This aspect of congestion losses will be addressed later in this section. For presentation of the basic model, we assume here that there is no upper bound on the values that the window can take.
} 
process depends only on the current window size $x_{t}$ of the connection. Let $\lambda(x)$ be the rate of $N(t)$ process when window size is $x_{t}=x$. Each loss results in a window reduction (this is because TCP assumes that each packet drop/mark corresponds to a congestion event in the network). Under the fluid model, it is standard to assume that this window reduction is reflected as an instantaneous jump in the $x_{t}$ process. The assumptions imply that $P\{N(t+\Delta)-N(t)=1\}=1-P\{N(t+\Delta)-N(t)=0\}=\lambda\left(x_{t}\right) \Delta+o(\Delta)$. Thus for small $\Delta$, if $N(t+\Delta)-N(t)=1$, the window is instantaneously reduced as

$$
x_{t+\Delta}=g\left(x_{t}\right)+o(\Delta),
$$

for some function $g(\cdot)$ such that $g(x)<x$ and $g\left(x_{\min }\right)=x_{\min }$. We assume that $g(\cdot)$ is such that if $x_{1}<x_{2}$ then either $g\left(x_{1}\right)<g\left(x_{2}\right)$ or $g\left(x_{1}\right)=g\left(x_{2}\right)=x_{\text {min }}$. This assumption implies that the set $s(x)=\{u \geq x: g(u) \leq x\}$ is connected. Define also $h(x)=\sup \{u \geq x: g(u) \leq$ $x\}=\sup s(x)$; we will also use the notation $g^{-1}(x)$ to mean $h(x)$.

\subsection{Transformation to an AWP with a Linear Increase Profile}

For a function $F(x)$ such that $\frac{d F(x)}{d x}=\frac{1}{f(x)}$, let us define a new process

$$
y_{t}=F\left(x_{t}\right) \text {. }
$$

Then the transformed process $\left\{y_{t}\right\}$ is such that, when the window $\left\{x_{t}\right\}$ is increasing, we have

$$
y_{t+\Delta}-y_{t}=\frac{x_{t+\Delta}-x_{t}}{f\left(x_{t}\right)}+o(\Delta)=\Delta+o(\Delta) \text {. }
$$

The reason for introducing this transformation is that it simplifies the analysis and visualisation of the window evolution process since now the transformed process has a linear increase profile $\left(y_{t+\Delta}-y_{t}=\Delta+o(\Delta)\right)$. Since $f(\cdot)>0$, it is seen that $F(\cdot)$ is strictly increasing and hence invertible. Thus there is a one to one correspondence between an AWP and its linearly increasing counterpart. A detailed justification of the above transformation is given in Appendix A. Under the above transformation, the loss process now has an intensity $\tilde{\lambda}(y) \triangleq \lambda\left(F^{-1}(y)\right)$. The decrease profile in case of a loss event in interval $[t, t+\Delta]$ of this transformed protocol will be determined by $F(\cdot)$ and $g(\cdot)$ as,

$$
y_{t+\Delta}=G\left(y_{t}\right)+o(\Delta)
$$

where $G(\cdot) \triangleq F\left(g\left(F^{-1}(\cdot)\right)\right)$ is assumed to have same properties as $g(\cdot)$.

The map $F: W \mapsto Y$ is actually a transformation from a general increase protocol to an additive increase protocol (like that of TCP's congestion avoidance algorithm). Thus it is enough to study protocols following additive increase general decrease algorithm. In the remaining of this section we will work only with AWP that has a linear increase profile and we need only consider the general decrease profile and a general window dependent loss rate $\lambda(\cdot)$. 
Let $y_{\text {min }} \triangleq F\left(x_{\text {min }}\right)$ be the lower bound on the transformed window size. Then $G\left(y_{\min }\right)=$ $y_{\min }$ Let

$$
\begin{array}{r}
S(y) \triangleq\{u \geq y: G(u) \leq y\}, \\
H(y) \triangleq \sup S(y) .
\end{array}
$$

Since the set $s(x)$ is connected and compact for each $x$ with inf $s(x)=x$, the set $S(y)$ is also connected and compact for any given $y$ and $\inf S(y)=y$. Note that the above definitions imply that

$$
G(H(y))=y .
$$

The interpretation of these quantities are as follows: $S(y)$ is the set of all possible window sizes, at least $y$, such that an occurrence of a loss event at these window sizes results in a window size of at most $y$ and $H(y)$ is the maximum such window size.

We now give the quantities defining an AWP and the transformation introduced above for some standard examples.

1. For the case of an additive increase multiplicative decrease protocol like the congestion avoidance algorithm of standard TCP, $f(x)=1, g(x)=\frac{x}{2}, F(x)=x, G(y)=\frac{y}{2}$, $S(y)=[y, 2 y]$ and $H(y)=2 y$.

2. For the case of a multiplicative increase multiplicative decrease protocol like the slow start algorithm of standard TCP or the Scalable TCP [8], $f(x)=\alpha x, g(x)=\beta x$, $F(x)=\frac{\log (x)}{\alpha}, G(y)=y-\theta, S(y)=[y, y+\theta]$ and $H(y)=y+\theta$, for some $\alpha>0, \beta<1$ and $\theta=-\frac{\log \beta}{\alpha}$.

\subsection{Incorporating a Bound on the Window Size}

The window evolution process described above does not incorporate any bound on the maximum allowed window size. In practice however there will be an upper bound $M$ on the window size that the AWP is allowed to use. This bound usually is either the receiver's advertised window (which is the maximum number of packets that the receiving entity's receive buffer can accommodate) or the total round trip pipe size. The behavior of the AWP under these two bounds are very different. In the first case where the window is restricted by the receiver's advertised window $M$, the window size stays at this value till another loss event takes place. While in the case where $M$ represents the round trip pipe size, reaching this limit results in an instantaneous congestion loss and the window size is reduced. However since the loss rate is assumed to be function of window size alone, it follows that we can study the second case via the first case (for details, see [5] which also addresses this issue for a constant loss rate). Hence in what follows we will restrict ourselves to the case where $M$ represents the window limitation owing to the receiver's advertised window.

Assume that the range of the values of the window process is divided into the intervals between points $\left[H^{j}\left(y_{\min }\right), H^{j+1}\left(y_{\min }\right)\right]$ where $H^{j}$ is $j$-fold composition of $H(\cdot)$ with 


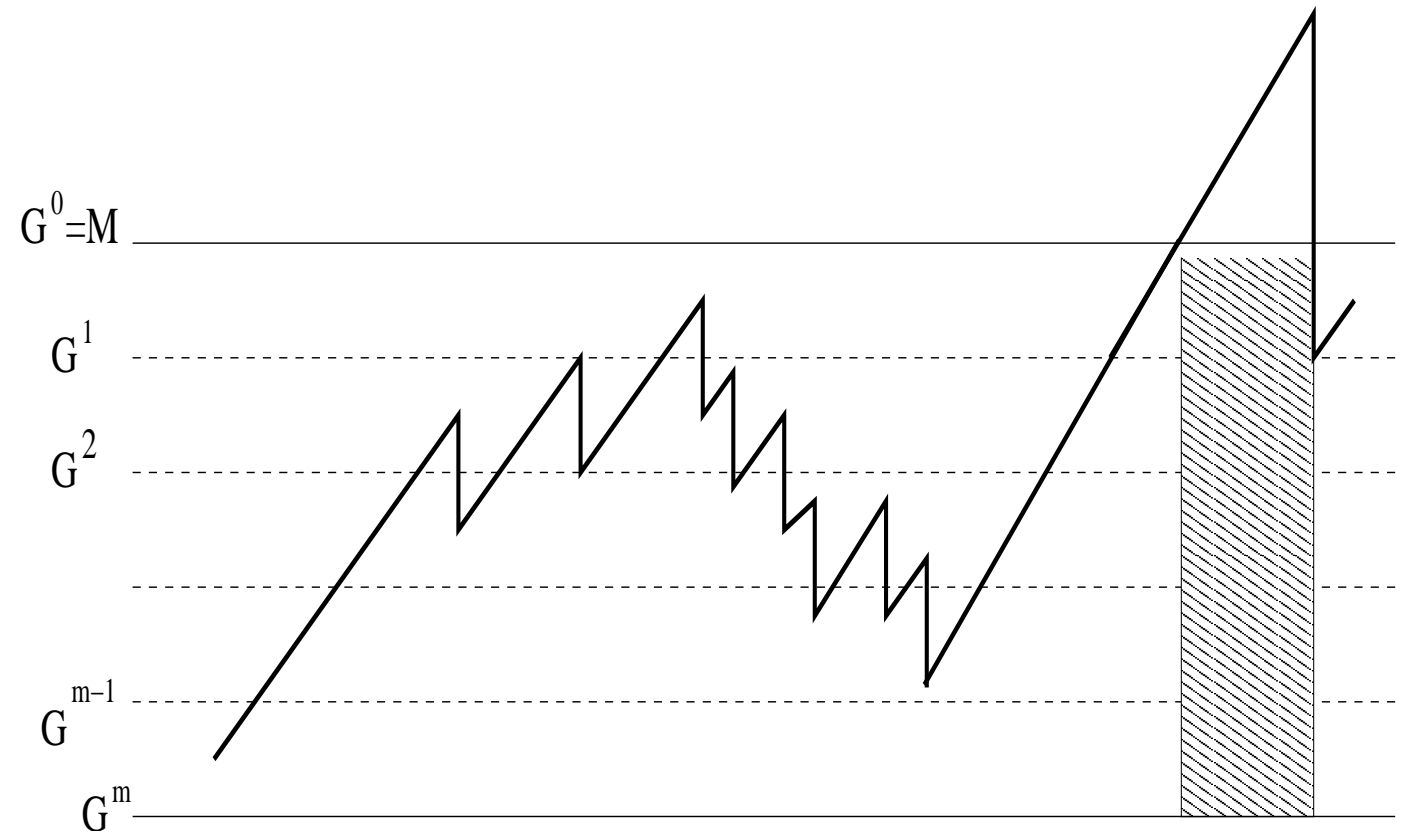

Figure 1: Evolution of the transformed window process $\left\{y_{t}\right\}$.

$H^{0}\left(y_{\text {min }}\right) \triangleq y_{\text {min }}$. Consider an $M$ such that $M=H^{m}\left(y_{\text {min }}\right)$ for some $m \geq 1$. Note that, under our choice of $M, H^{j}\left(y_{\text {min }}\right)=G^{m-j}(M)$ with $G^{0} \triangleq M$ and $G^{i}=G\left(G^{i-1}\right)$. Let $l_{i} \triangleq G^{i-1}-G^{i}$. Under the above definitions, $y \in\left[G^{i}, G^{i-1}\right] \Rightarrow H(y) \in\left[G^{i-1}, G^{i-2}\right]$. The case where such an $m$ does not exist, i.e., $H^{m-1}<M<H^{m}$ for some $m$, is not possible since the definition of $G(\cdot)$ depends on $y_{\min }$ and $M$ implicitly, and it ensures that $G\left(G^{m-1}\right)=y_{\min }$ so that $H^{m}=M$.

We consider a further modification in the evolution of the window process $y_{t}$; this is shown in Figure 1. For this modified process, the window size is unbounded. However, when $y>G^{0}$, we assume that the loss rate is constant and equal to $\lambda\left(G^{0}\right)$. We also assume that if a loss event takes place when $y \geq G^{0}$, the window is dropped to $G^{1}=G(M)=H^{m-1}\left(y_{\text {min }}\right)$. The evolution of the modified process for $y<G^{0}$ is unchanged, i.e., a loss event occurs with rate $\lambda\left(y_{t}\right)$ and the window is dropped to $G\left(y_{t}\right)$ in case of a loss event when $y_{t}<G^{0}$. Note that we are assuming a linear increase of $y_{t}$ for any value of $y$. Thus, the modified process has the following evolution: the increase profile is given by

$$
y_{t+\Delta}=y_{t}+\Delta+o(\Delta)
$$


Losses occur according to a Poisson process of rate $\lambda\left(y_{t} \wedge G^{0}\right)$ and the window reduction in case of a loss event in time interval $(t, t+\Delta)$ is

$$
y_{t+\Delta}=G\left(y_{t} \wedge G^{0}\right) .
$$

Remark If the window size is bounded (as the case will be in the rest of this paper), so is $\lambda(\cdot)$. In this scenario, it is sometimes convenient to assume that the process $N(t)$ is actually derived from a standard Poisson process $\Lambda(t)$ of unit rate so that a jump in $\Lambda(t)$ results in a jump in $N(t)$ only with probability $\frac{\lambda\left(x_{t}\right)}{\sup _{x} \lambda(x)}$. A similar, though not same, approach will be used in Section 4 to obtain stationary distribution for a general loss rate and in Section 8 to establish a necessary and sufficient condition for stability of the window process.

\subsection{Performance Measure}

There can be various performance measures of interest in the context of the problem under consideration. Most prominent of these (and the one most frequently used in literature) is the expected window size. However, finding only expected window size may not give much information about the window process itself. An analysis for the performance of an AWP should also consider the stationary window size distribution. In this paper we are interested in obtaining the stationary window size distribution of the AWP.

\subsection{The Kolmogorov Equations}

Let $\pi(y)$ be the density function and $\Pi(y)$ be the distribution function of the (modified) $y_{t}$ process (note that we are suppressing the dependence on $M$ here.).

Let, for a fixed $t,[t, t+\Delta]$ be a small time interval. When the process is in equilibrium, the probability of upcrossing level $y$ during $[t, t+\Delta]$ is

$$
P\left\{y_{t} \in(y-\Delta, y)\right\} P\{\text { no loss during }[t, t+\Delta]\}=\pi(y) \Delta(1-\lambda(y) \Delta)+o(\Delta)
$$

Similarly, the probability of downcrossings is

$$
\begin{gathered}
\int_{u=y}^{H(y)} P\{Y \in(u, u+d u)\} \lambda(u) \Delta=\int_{u=y}^{H(y)} \pi(u) \lambda(u) d u \Delta+o(\Delta) \quad y<G^{1}, \\
\int_{u=y}^{\infty} P\{Y \in(u, u+d u)\} \lambda(u) \Delta=\int_{u=y}^{\infty} \pi(u) \lambda(u) d u \Delta+o(\Delta) \quad G^{1}<y \leq G^{0}, \\
\int_{u=y}^{\infty} P\{Y \in(u, u+d u)\} \lambda\left(G^{0}\right) \Delta=\int_{u=y}^{\infty} \pi(u) \lambda\left(G^{0}\right) d u \Delta+o(\Delta) \quad y \geq G^{0} .
\end{gathered}
$$

In the steady state, the probability of up-crossing is equal to that of down-crossing. Thus, letting $\Delta \rightarrow 0$, we obtain

$$
\pi(y)= \begin{cases}\int_{u=y}^{\infty} \pi(u) \lambda\left(G^{0}\right) d u=\lambda\left(G^{0}\right) \Pi(y), & y \geq G^{0} \\ \int_{u=y}^{G} \pi(u) \lambda(u) d u+\lambda\left(G^{0}\right) \Pi^{c}\left(G^{0}\right), & G^{1}<y \leq G^{0} \\ \int_{u=y}^{H}(y) & y_{\min } \leq y<G^{1}\end{cases}
$$

INRIA 
Using integrating factor method for the Kolmogorov equation for $y \geq G^{0}$,

$$
\Pi(y)=\Pi\left(G^{0}\right) e^{-\lambda\left(G^{0}\right)\left(y-G^{0}\right)}, \quad y \geq G^{0} .
$$

The basic idea involved in solving the Kolmogorov equations obtained above is to use the knowledge of $\lambda(\cdot)$ to obtain $\pi(y)$ for $y \in\left[G^{1}, G^{0}\right]$. Now, one can find $\pi(y)$ for $y \in\left[G^{i+1}, G^{i}\right]$ from the knowledge of $\pi(y)$ for $y \in\left[G^{i}, G^{i-1}\right]$. In this process, since we need to integrate over different regions, integration constants appear naturally. These integration constants are computed using continuity of $\Pi(\cdot)$ at the boundaries $G^{i}$. Clearly, the form of $\pi(\cdot)$ will depend on that of $\lambda(\cdot)$ and of $H(\cdot)$.

Remark For the window evolution with an increase profile $f(\cdot)$, decrease profile $g(\cdot)$ and loss rate $\lambda(\cdot)$, the Kolmogorov equations are

$$
f(x) \pi(x)= \begin{cases}\int_{u=x}^{\infty} \pi(u) \lambda\left(G^{0}\right) d u=\lambda\left(G^{0}\right) \Pi(x), & x \geq G^{0}, \\ \int_{u=x}^{G^{0}} \pi(u) \lambda(u) d u+\lambda\left(G^{0}\right) \Pi^{c}\left(G^{0}\right), & G^{1}<x \leq G^{0}, \\ \int_{u=x}^{H(x)} \pi(u) \lambda(u) d u, & x_{\text {min }} \leq x<G^{1} .\end{cases}
$$

\subsection{Connection to Piecewise Deterministic Markov Processes}

We briefly mention that the real valued process $\left\{x_{t}\right\}$ above is actually a special instance of a family of stochastic processes called the piecewise deterministic Markov process [21, 22]. The PDMPs are allowed to take values in more general spaces. A PDMP is determined by the (possibly state dependent) jump rates and a deterministic evolution between these jumps. A special instance of a possible evolution of such a process is given in Figure 2. Here the process $x_{t}$ is shown to be piecewise linear as is the case with $\left\{y_{t}\right\}$ process above ${ }^{2}$. A downward jump occurs according to a Poisson process of rate $\lambda\left(x_{t}\right)$. The additional generality in the case of PDMPs is that it allows for a random amount of jump whose distribution itself can depend on $x_{t}$; we let $B(x, y)$ denote the probability that a jump is to point less than or equal to $y$ given that a downward jump occurred when $x_{t}=x$. In the running example of evolution of the window process $\left\{x_{t}\right\}$, we see that $B(x, \cdot)$ is a unit step function since the jumps are deterministic, i.e., $B(x, y)=I\{y>g(x)\}$.

\section{Relations between Two Systems of Window Evolution}

We now consider two systems, 1 and 2, having their own increase and decrease profiles and loss rates, denoted $f_{i}(\cdot), g_{i}(\cdot), \lambda_{i}(\cdot), i \in\{1,2\}$. We provide a condition under which these two systems have related stationary probability distribution. Assuming that $g_{1}(x)=$ $g_{2}(x)=g(x), \forall x$, and that in both the systems the upper bound on the window is same

\footnotetext{
${ }^{2}$ In general, if the deterministic evolution between jumps is not linear but given by a (here one dimensional) drift that depends only on $x_{t}$ then it may be transformed to a linear increase in a similar way as we obtained the $\left\{y_{t}\right\}$ process from $\left\{x_{t}\right\}$ process in Section 2.1 .
}

$\mathrm{RR} \mathrm{n}^{\circ} 5262$ 


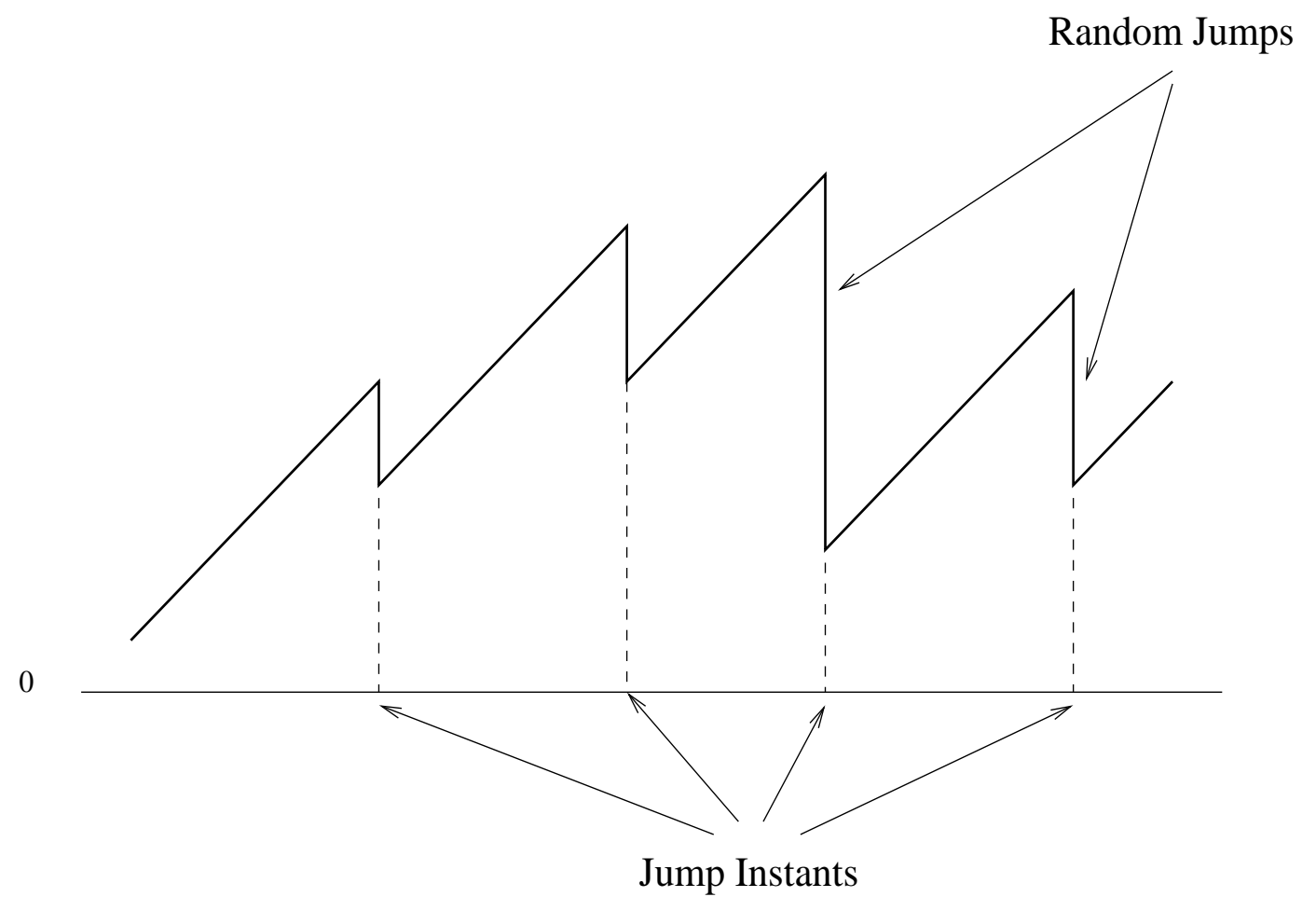

Figure 2: An instance of evolution of a PDMP.

(and is equal to $M$ ), the Kolmogorov equations for the two systems are

$$
\begin{aligned}
f_{i}(x) \pi_{i}(x) & =\int_{u=x}^{g^{-1}(x)} \lambda_{i}(u) \pi_{i}(u) d u \\
\frac{f_{i}(x)}{\lambda_{i}(x)} \frac{\lambda_{i}(x) \pi_{i}(x)}{E\left[\lambda_{i}(X)\right]} & =\int_{u=x}^{g^{-1}(x)} \frac{\lambda_{i}(u) \pi_{i}(u)}{E\left[\lambda_{i}(X)\right]} d u
\end{aligned}
$$

where $E\left[\lambda_{i}(X)\right]=\int_{x} \lambda_{i}(x) \pi_{i}(x) d x$ is the expected loss rate in $i^{t h}$ system. It is clear from the above set of equations that if $\frac{f_{1}(x)}{\lambda_{1}(x)}=\frac{f_{2}(x)}{\lambda_{2}(x)}, \forall x$, the functions $\frac{\lambda_{1}(x) \pi_{1}(x)}{E\left[\lambda_{1}(X)\right]}$ and $\frac{\lambda_{2}(x) \pi_{2}(x)}{E\left[\lambda_{2}(X)\right]}$, both being probability density functions integrating to unity, are equal for each $x$. Thus,

Theorem 1 If two AWP controlled window evolution are such that both have same drop profile and have the same ratio of increase profile and loss rate for each $x$, then

$$
\frac{\pi_{1}(x)}{\pi_{2}(x)}=C \frac{\lambda_{2}(x)}{\lambda_{1}(x)}=C \frac{f_{2}(x)}{f_{1}(x)}
$$


where $C=\frac{E\left[\lambda_{1}(X)\right]}{E\left[\lambda_{2}(X)\right]}$.

This result is important as it gives us a way to analyse one system using the analysis of the other related system. We use this result in Section 6.2 where we use the observation that an AIMD protocol with constant loss rate and an MIMD protocol with linear loss rate satisfy the requirement of Theorem 1 as for the first (AIMD) system $f(x)=\alpha$ and $\lambda(x)=\lambda$ while for the second (MIMD) system $f(x)=\alpha x$ and $\lambda(x)=\lambda x$ and both have same multiplicative decrease factor. Since the analysis for the first system is known from [5], we use it to find stationary distribution for the MIMD protocol with linear loss rate. See Section 6.2 for details.

In the special case where both the system use multiplicative decrease profile with a constant decrease factor $\beta$, we can get some more detailed equivalence between two related systems. This is done next.

\subsection{A Queueing Model for Multiplicative Decrease Protocols}

Consider an AWP with a constant multiplicative decrease factor $\beta$. Introduce the transformation $z_{t}=\ln M-\ln x_{t}$ (note that we are performing the transformation on the original window process $x_{t}$ and not on the transformed process $y_{t}$ that has a linear increase profile). Note that we are assuming that $z_{t}$ is unbounded, i.e., that $x_{m i n}=0$; we can do this since we can use standard approach ([3, Chapter 14]) to analyse the case where $z_{t}$ is bounded by $\ln M-\ln x_{m i n}$ from that where $z_{t}$ is unbounded. The evolution of the process $z_{t}$ now is as shown in Figure 3. It is evident from the transformation (as also visualised in the figure), the multiplicative decrease of the process $x_{t}$ presents itself as a constant increase of $\ln \beta$ in the evolution of $z_{t}$ process. The evolution of $z_{t}$ process suggests that $z_{t}$ can be thought of as workload process of a queue for which the service requirement of the customers is constant $(-\ln \beta)$. If the increase profile and loss rate for $x_{t}$ process are $f(\cdot)$ and $\lambda(\cdot)$, then

in the $z_{t}$ process, the customer arrival rate is $\lambda\left(M e^{-z_{t}}\right)$ and service rate is $\frac{f\left(M e^{-z_{t}}\right)}{M e^{-z_{t}}}$, both depending on the workload process $z_{t}$. Thus we get a queueing system with constant service requirements and state dependent service rates and arrival rates. This observation leads us to following

Theorem 2 Consider window evolutions in the two systems 1 and 2 introduced above, both with same multiplicative decrease profile. If $\frac{f_{1}(x)}{\lambda_{1}(x)}=\frac{f_{2}(x)}{\lambda_{2}(x)}$ then the distribution of window size just before loss instants is same in both the systems.

Proof The logarithmic transformation introduced above maps the two system into queueing systems with constant service requirements. The proof then follows from [16, Theorem 3.3] which says that for two queueing systems with same service requirement distribution, if the ratio of the two arrival rates is same as that of their service rates for any workload, then the stationary distribution of the workload process seen just before an arrival is same for both the system. The proof follows from the relation between the loss rate in window process and the arrival rate in the queueing system and that between the increase profile in the window process and the service rate in the queueing system.

$\mathrm{RR} \mathrm{n}^{\circ} 5262$ 
$\mathbf{M}$

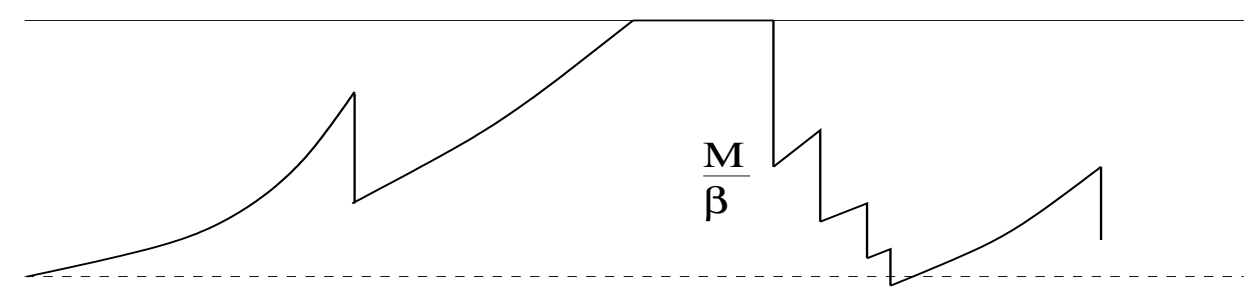

$\ln \mathrm{M}$

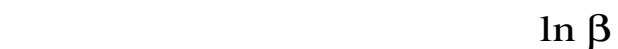

$\ln \beta$

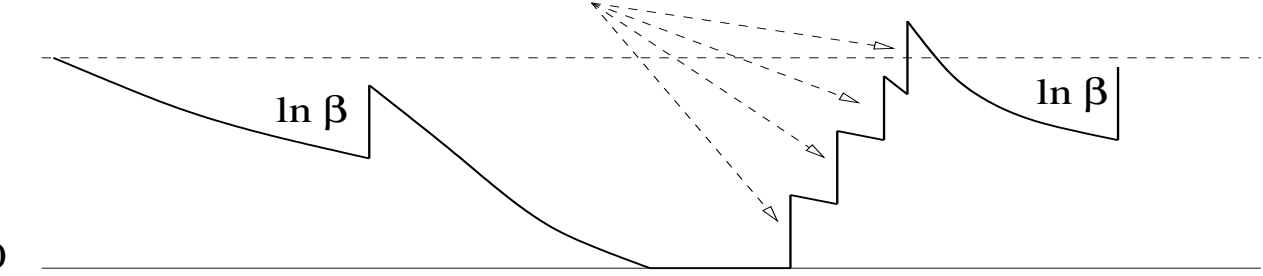

Figure 3: The original window evolution (top) and its transformation to the workload process in a queue (bottom).

Applying this result to the two systems satisfying the above condition where the first one is AIMD with constant loss rate and the second one is MIMD with linear loss rate, we see that the stationary distribution of the window process just before (and hence just after) loss instants is same. Thus, the standard AIMD protocol with constant loss rate is same as MIMD protocol with linear loss rate in the sense that the distribution of the window sizes just before losses are the same for the two.

Further, since Theorem 2 is valid for any two AWPs satisfying the required conditions, it is seen that if for one of the AWPs the loss rate is constant, the PASTA property implies that the stationary (time average) distribution of the window size in the system with constant loss rate is same as the window size distribution just before losses in either of the system.

Now we specialize Theorem 1 to the case of multiplicative decrease protocols and provide a stronger result.

Theorem 3 Consider window evolutions in the two systems 1 and 2 introduced above, both with same multiplicative decrease profile. If $\frac{f_{1}(x)}{\lambda_{1}(x)}=\frac{f_{2}(x)}{\lambda_{2}(x)}$ then the time average distribution of window size $\pi_{i}(\cdot)$ in the two systems is related by

$$
\frac{\pi_{1}(x)}{\pi_{2}(x)}=C \frac{f_{2}(x)}{f_{1}(x)}=C \frac{\lambda_{2}(x)}{\lambda_{1}(x)}
$$

where $C=\frac{\lambda_{1}(M) \Pi_{1}^{c}(M)}{\lambda_{2}(M) \Pi_{2}^{c}(M)}$ with $\Pi_{i}^{c}(\cdot)$ denoting the complementary distribution function. 
Proof Follows from [16, Theorem 3.1] which states that the two queueing systems if the ratio of the two arrival rates is same as that of their service rates for any workload, then the density corresponding to the time average stationary distribution of the two systems, $\pi_{1}(z)$ and $\pi_{2}(z)$ are such that $\pi_{1}(z) r_{2}(z)=C \pi_{2}(z) r_{1}(z)$ where $r_{i}(\cdot)$ is the service rate in the $i^{t h}$ system and $C=\frac{\Pi_{1}(0)}{\Pi_{2}(0)}$. The proof follows from the relation between the loss rate in window process and the arrival rate in the queueing system and that between the increase profile in the window process and the service rate in the queueing system.

Corollary 1 Consider the scenario of Theorem 3. Then

$$
\frac{E\left[\lambda_{1}(X)\right]}{E\left[\lambda_{2}(X)\right]}=\frac{\lambda_{1}(M) \Pi_{1}^{c}(M)}{\lambda_{2}(M) \Pi_{2}^{c}(M)}
$$

Proof Follows from Theorem 1 and Theorem 3.

Application of this result to the running example of the two systems where the first one is AIMD with constant loss rate and other is MIMD with linear loss rate, we see that the expected window size in the MIMD case is $E[X]=\frac{M \Pi_{2}^{c}(M)}{\Pi_{1}^{c}(M)}$.

Remark It is important to note that the window process with a lower bound of 1 and an upper bound of $M<\infty$ is always ergodic in the case of multiplicative decrease algorithm. This is because for any bounded loss rate and positive increase profile, the window process $\left\{x_{t}\right\}$ is irreducible. However, if we assume $x_{\min }=0$, then the corresponding unbounded transformed queueing process need not always be ergodic. Thus, we can not always use the truncation method of [3] mentioned above. Hence it becomes necessary to solve the detailed Kolmogorov equations for each case. This remark is, in particular, relevant for the case where the AWP follows a multiplicative increase multiplicative decrease algorithm and the loss rate is constant. For this case the transformed process $z_{t}$ is just the workload process of an $\mathrm{M} / \mathrm{D} / 1$ queue. However we can not use this approach for $\lambda>-\ln \beta$ owing to the above mentioned reason.

Remark The process $z_{t} \triangleq M-x_{t}$ always represents the workload process in a queue with state dependent arrival rate, service rate and service requirement.

Remark The results of this section indicate that if the losses come from an AQM scheme, then there are many AWP-AQM pairs (i.e., $f(\cdot)$ and $\lambda(\cdot)$ ) which have the same drop profile $(g(\cdot))$ and have similar performance (in the sense of Theorem 1). Moreover, if the decrease profile is fixed to be a multiplicative one, we see that all these AWP-AQM pairs have same window distribution before drop instants (Theorem 2).

Having made the relation between the evolution of the window process of the AWP and the workload process in a queueing system, we now proceed to solve the Kolmogorov equations considering specific forms of the $\lambda(\cdot)$ and $H(\cdot)$ functions. As remarked above, analysing the queueing system does not provide us with the stationary distribution for all the possible values of the involved parameters. This makes it necessary to solve the Kolmogorov equations for each instance of the problem. First we give a solution of the Kolmogorov equation (upto a multiplicative constant) for a general AWP with a general loss rate.

$\mathrm{RR} \mathrm{n}^{\circ} 5262$ 


\section{Solution to the Kolmogorov Equations}

The general Kolmogorov equation for the stationary distribution of an AWP with increase profile $f(\cdot)$, decrease profile $g(\cdot)$ and loss rate $\lambda(\cdot)$ is

$$
\begin{aligned}
f(x) \pi(x) & =\int_{u=x}^{g^{-1}(x)} \lambda(u) \pi(u) d u \\
\frac{f(x)}{\lambda(x)} \frac{\lambda(x) \pi(x)}{E[\lambda(X)]} & =\int_{u=x}^{g^{-1}(x)} \frac{\lambda(u) \pi(u)}{E[\lambda(X)]} d u,
\end{aligned}
$$

where $E[\lambda(X)]=\int_{x=x_{m i n}}^{\infty} \lambda(x) \pi(x) d x$. Note that $E[\lambda(X)]$ always exists if the window process is bounded by a quantity $M$. Introducing the transformation $\tilde{\pi}(x)=\frac{\lambda(x) \pi(x)}{E[\lambda(X)]}$, we get

$$
\frac{f(x)}{\lambda(x)} \tilde{\pi}(x)=\int_{u=x}^{g^{-1}(x)} \tilde{\pi}(u) d u
$$

which is the Kolmogorov equation of an AWP whose increase profile is $\frac{f(x)}{\lambda(x)}$ and decrease profile is $g(x)$ while the loss rate now is a state-independent constant, equal to unity; We will see an example of such an approach in Section 6.2. We can further introduce a transformation of the new protocol (having increase profile $\frac{f(x)}{\lambda(x)}$ ) to another AWP with a linear increase profile as indicated in Section 2.1. Thus, without loss of generality, we can assume that the protocol under consideration has a linear increase profile and the loss rate is unity. Now we provide an expression for the stationary probability distribution for the bounded process with linear increase, a unit loss rate and a general decrease profile $G(\cdot)$.

Theorem 4 For $M>x>G^{1}$

$$
e^{x} \Pi^{c}(x)=c_{1},
$$

For $x \in I_{k}=\left[G^{k}, G^{k-1}\right], k \geq 2$,

$$
e^{x} \Pi^{c}(x)=\sum_{j=1}^{k} c_{j} J_{k, k-j}(x)
$$

And

$$
P_{M} e^{M}=c_{1}=\left[\sum_{j_{1}=1}^{m-1} \sum_{j_{2}=1}^{j_{1}-1} \ldots \sum_{j_{m-2}=1}^{j_{m-3}-1} q_{m, j_{1}} q_{j_{1}, j_{2}} \ldots q_{j_{m-2}, 1}\right]^{-1}
$$

where $c_{k}=e^{G^{k}} \Pi^{c}\left(G^{k}\right)$, the constants $q_{k, j}$ are defined as $q_{k, j}=\left[J_{k-1, k-1-j}\left(G^{k-1}\right)-\right.$ $\left.J_{k, k-j}\left(G^{k-1}\right)\right]$, with $\int_{u=G^{2}}^{x} e^{-D(u)} d u=J_{2,1}(x)$, and $J_{k, l}(x)=\int_{G^{k}}^{x} e^{-D(x)} J_{k-1, l-1}(H(u)) d u$ (for $x \in I_{k}$ ) and $D(x)=H(x)-x$ is the downward jump at $H(x)$ and $M=H^{m}\left(x_{\text {min }}\right)$.

INRIA 
Proof See Appendix B.

Remark It is worth noting that Theorem 4 gives the workload process distribution in a queue with Poisson arrival process, state dependent deterministic service requirements and bounded workload process (this is because the process $M-x_{t}$ corresponds to the workload process in the mentioned queueing system). In Proposition 2 we give details for the standard $\mathrm{M} / \mathrm{D} / 1$ queue with finite workload capacity.

Remark As mentioned in the beginning of this section, the stationary distribution for the transformed system of AWP with linear increase profile and constant loss rate with a bounded window also gives the distribution of the original window process with state dependent loss rate and general increase profile upto a multiplicative constant. Hence Theorem 4 gives the solution to the Kolmogorov equation for a general AWP with a general loss rate (upto a multiplicative constant of $E[\lambda(X)]$ ).

Till now the development did not consider exact form of loss rate $\lambda(\cdot)$ for the original process. In the following sections we consider specific forms of $\lambda(\cdot)$ to find the stationary window size distribution and work out the solution of Kolmogorov equation for several available TCP versions. We start with the case where $\lambda(x) \equiv \lambda$, independent of the current window size in Section 5 . We then consider the situation of a linearly increasing loss rate, i.e., $\lambda(x)=\lambda x$ in Section 6 .

We remark here that a linear loss rate, $\lambda(x)=\lambda x$ is suitable for the cases where, like NewReno version of TCP, only one window reduction takes place irrespective of the number of losses in a round-trip time and each packet is dropped with a fixed probability.

\section{Constant Loss Rates: $\lambda(x)=\lambda$}

In this section we first present the Kolmogorov equations for the case where the loss rate is constant, independent of the window size process and then we give a method of solving the Kolmogorov equations for a general AWP.

First observe that any transformation applied to the window size does not affect the loss rate. Thus for any given AWP, we can always apply the transformation introduced in Section 2.1 to get a linear increase profile. For the evolution of this transformed process, we see that the jump rate (loss rate) is still $\lambda$, independent of anything else. Thus we need only study the case of linear increase general decrease protocols. In this section we first identify the special structure of the Kolmogorov equation for window evolution with constant loss rate with a general decrease profile. We then work out the details by fixing a particular drop profile, i.e., multiplicative decrease as in standard congestion avoidance algorithm of TCP.

Here we do not dwell into the issue of lower bound $x_{\min }$ on the window size of the original process. This is because the lower bound on the transformed process is $y_{\min }=F\left(x_{\min }\right)$ can take very different values depending on $F(\cdot)$. For example, if the original AWP is multiplicative increase multiplicative decrease (MIMD), the function $F(\cdot)$ turns out to be logarithmic and hence the lower bound $y_{\min }$ can be $-\infty$ or 0 depending on whether $x_{\min }=0$ or 1 , respectively.

$\mathrm{RR} \mathrm{n}^{\circ} 5262$ 
For this case the Kolmogorov equations can be written as

$$
\begin{aligned}
& \pi(y)=\int_{u=y}^{\infty} \pi(u) \lambda d u, \quad G^{1}<y, \\
& \pi(y)=\int_{u=y}^{H(y)} \pi(u) \lambda d u, \quad y_{\min } \leq y<G^{1} .
\end{aligned}
$$

Using integrating factor method for the Kolmogorov equation for $y \geq G^{1}$,

$$
\Pi(y)=\Pi\left(G^{1}\right) e^{-\lambda\left(y-G^{1}\right)}, \quad y \geq G^{1} .
$$

For $x \in\left[G^{k}, G^{k-1}\right], k \geq 1$, let $\Pi_{k}(x) \triangleq \Pi(x)$. Thus, for $k \geq 2$,

$$
\frac{d}{d y} \Pi_{k}(y)=\Pi(H(y)) \lambda-\Pi(y) \lambda .
$$

Assuming that $H(\cdot)$ are such that $\Pi(\cdot)$ is continuous at $G^{i}, \forall i$, we have

$$
\Pi_{k}\left(G^{k-1}\right)=\Pi_{k-1}\left(G^{k-1}\right), \quad k \geq 2 .
$$

This gives us $\Pi_{k}(\cdot)$ recursively as

$$
\Pi_{k}(x)=\Pi_{k-1}\left(G^{k-1}\right) e^{\lambda\left(G^{k-1}-x\right)}-\lambda e^{-\lambda x} \int_{u=x}^{G^{k-1}} e^{\lambda u} \Pi_{k-1}(H(u)) d u, \quad k \geq 2 .
$$

Similar approach has also been used in [5] which considers an AIMD protocol with constant loss rate. The above system of equations will be solved and used in Section 8. Here we obtain the stationary distribution for the case of an MIMD protocol (like Scalable TCP [8]) with constant loss rate.

\subsection{Application to MIMD Protocols with Bounded Window}

Once again our approach will be to transform the MIMD window evolution to the case of a linear increase profile. For the case of MIMD protocols, the window evolution is described as follows. In case of no loss in interval $[t, t+\Delta]$, the window increases to

$$
x_{t+\Delta}=\left(x_{t}+\alpha x_{t} \Delta+o(\Delta)\right) \wedge M,
$$

for some $\alpha>0$ and an upper bound $M$ on the window size. In case of a loss in interval $[t, t+\Delta]$, the window decreases to

$$
x_{t+\Delta}=\left(\beta x_{t}\right) \vee 1+o(\Delta),
$$

where $1>\beta>0$ is the multiplicative decrease constant. The natural lower bound of $x_{t} \geq 1$ packet applies. It is clear now that the transformation $x_{t} \mapsto \frac{\log x_{t}}{\alpha} \triangleq y_{t}$ results in the process 
$\left\{y_{t}\right\}$ having linear increase profile. The transformed window after a loss event in interval $[t, t+\Delta]$ is given by

$$
y_{t+\Delta}=\left(y_{t}-\theta\right)^{+}+o(\Delta)
$$

where $\theta \triangleq \frac{-\log (\beta)}{\alpha}>0$.

Thus for this case, $y_{\min }=0$ and $H(y)=y+\theta$ Thus, from the construction of the (virtually) bounded process of Section $2, G^{0}=M=m \theta$ and $G^{l}=(m-l) \theta, G(u)=(u-\theta)^{+}$. This system has simple up and down crossing rates for $0 \leq y \leq(m-1) \theta$,

$$
\begin{aligned}
\pi(y) & =\lambda \int_{u=y}^{y+\theta} \pi(u) d u, \\
\Pi^{\prime}(y) & =\lambda(\Pi(y+\theta)-\Pi(y)),
\end{aligned}
$$

where $\pi(\cdot)$ and $\Pi(\cdot)$ are the density and distribution functions respectively for the $\left\{y_{t}\right\}$ process. Defining, for convenience, $\Pi_{k}(y)=\Pi(k \theta+y)$ for $0 \leq y \leq \theta$, we have

Proposition 1 For $0 \leq k \leq m-1$ and $0 \leq x \leq \theta$,

$$
\Pi_{k}^{c}(x)=\sum_{j=0}^{m-k-1} \Pi_{k+j}^{c}(0) \frac{(\lambda x)^{j}}{j !} .
$$

Proof: See Appendix C.

Proposition 2 The constants $\Pi_{k}^{c}(0)$ are given by

$$
\begin{aligned}
\Pi_{m-1}^{c}(0)= & {\left[\left(a^{m-1}-\phi_{1}(m-1)\right)+\sum_{s=1}^{m-3}(-1)^{s} \sum_{l=s}^{m-2} \phi_{s}(l)\left(a^{m-l-1}-\phi_{1}(m-l-1)\right)+\right.} \\
& \left.(-1)^{m-2}(a-b) \phi_{m-2}(m-2)\right]^{-1}
\end{aligned}
$$

and for $0 \leq k \leq m-2$,

$$
\begin{aligned}
\Pi_{k}^{c}(0)= & \Pi_{m-1}^{c}(0)\left[\left(a^{m-k-1}-\phi_{1}(m-k-1)\right)+(-1)^{m-k-2}(a-b) \phi_{m-k-2}(m-k-2)\right. \\
& \left.+\sum_{s=1}^{m-k-3}(-1)^{s} \sum_{l=s}^{m-k-2} \phi_{s}(l)\left(a^{m-k-l-1}-\phi_{1}(m-k-l-1)\right)\right] .
\end{aligned}
$$

with $a=e^{\lambda \theta}$ and $\phi_{j}(l)$ defined recursively as, $\phi_{0}(0)=0$ and

$$
\phi_{j+1}(l)=\sum_{s=1}^{l-j} \phi_{1}(s) \phi_{j}(l-s), \quad j \geq 1 .
$$

$\mathrm{RR} \mathrm{n}^{\circ} 5262$ 
Proof: See Appendix D.

Remark For the above case where $x_{\min }=1$, the evolution of process $\frac{\log M}{\alpha}-\frac{\log x_{t}}{\alpha}=$ $\frac{\log M}{\alpha}-y_{t}$ corresponds to the workload process of an $\mathrm{M} / \mathrm{D} / 1$ queue with a bounded workload capacity of $\frac{\log M}{\alpha}$ and service requirement of $\theta$ for each customer. This is a system similar to that of [17] with a difference that the model of [17] assumes that the customer that can make the workload to exceed a certain fixed threshold is lost. While in our case such a customer is not completely lost but is admitted with a service that makes the workload process equal to the threshold. Thus the above result is of independent interest in queueing theory.

Remark We can also easily incorporate another value of $0<x_{\min } \neq 1$ in the above analysis. As mentioned in Section 3.1, if we assume that $x_{\min }=0$, the transformation $\frac{\log M}{\alpha}-\frac{\log x_{t}}{\alpha}$ corresponds to the workload process of a classical M/D/1 queue. For this case the moments and the stationary window size distribution are well known.

\subsubsection{MIMD with Unbounded Window: A D/M/1 Queue}

Assuming that $M=\infty$, i.e., there is no bound on the window size, we can not use the results from above directly in this case. Another approach to obtain the stationary distribution $\Pi(\cdot)$ is to look at the process $\left\{y_{n}, n \geq 0\right\}$ embedded just after the loss instants of the transformed process with linear increase profile, $\left\{y_{t}\right\}$. Let $\left\{a_{n}, n \geq 0\right\}$ denote the time between two successive losses. Then, $\left\{y_{n}\right\}$ is a continuous state space Markov chain which is given by the recursive equation

$$
y_{n+1}=\left(y_{n}+a_{n}-\theta\right)^{+} .
$$

We note that the loss process $a_{n}$ is exponentially distributed with rate $\lambda$. Equation 4 is the same as the recursive equation for the workload in a $D / M / 1$ queue with interarrival time $\theta$ and mean service time $\frac{1}{\lambda}$. The steady state distribution of $y, P\left(y_{n} \leq y\right)$ can be obtained as [28]

$$
P\left(y_{n}>y\right)=\left(1-\frac{s_{1}}{\lambda}\right) e^{-s_{1} y},
$$

where $s_{1}$ is the root of the equation $s+\lambda=\lambda e^{s / \theta}$ in $\operatorname{Re}(s)<0$. The stability condition for the workload process of this $\mathrm{D} / \mathrm{M} / 1$ queue (and, equivalently, for the window size process $\left.\left\{y_{t}\right\}\right)$ is $\theta>\frac{1}{\lambda}$.

In order to obtain the distribution at a random arrival instant, we note that the window size just before loss instant, $y_{n+1}^{-}$, is given by

$$
y_{n+1}^{-}=y_{n}+a_{n} .
$$

Since $a_{n} \mathrm{~s}$ are exponentially distributed with parameter $\lambda$,

$$
\begin{aligned}
P\left(y_{n+1}^{-}>y\right) & =\lambda \int_{0}^{\infty} P\left(y_{n}>y-a\right) e^{-\lambda a} d a \\
& =\lambda \int_{y}^{\infty} e^{-\lambda a} d a+\lambda \int_{0}^{y} P\left(y_{n}>y-a\right) e^{-\lambda a} d a
\end{aligned}
$$

INRIA 


$$
\begin{aligned}
& =e^{-\lambda y}+\lambda\left(1-\frac{s_{1}}{\lambda}\right) e^{-s_{1} y} \int_{0}^{y} e^{-\left(\lambda-s_{1}\right) a} d a \\
& =e^{-s_{1} y}
\end{aligned}
$$

Using PASTA property, the window size distribution at a random time is the same as that seen by the loss arrivals. Since $y=\frac{\log x}{\alpha}$, the window distribution at any random time is

$$
P\left(x_{t}>x\right)=x^{-\frac{s_{1}}{\alpha}}
$$

Remark This approach can also be used for bounded window process when loss rate is large enough so that the bound is attained with negligible probability.

Remark If the window size in the original process $\left\{x_{t}\right\}$ is bounded by a value of $M$ then the evolution of the process $\left\{y_{n}\right\}$ (now embedded just before loss instants in the process $\left.\left\{y_{t}\right\}\right)$ is

$$
y_{n+1}=\min \left(\left(y_{n}-\theta\right)^{+}+a_{n}, \frac{\log M}{\alpha}\right)
$$

which is the workload just after an arrival in a $\mathrm{D} / \mathrm{M} / 1$ queue with a bounded workload capacity of $\frac{\log M}{\alpha}$. The connection to $\mathrm{M} / \mathrm{D} / 1$ queue implies that this is also the residual workload seen by arriving customers in an $M / D / 1$ queue with finite workload capacity. We have, using the PASTA property in the M/D/1 system,

Theorem 5 The distribution of workload process just after arrivals in a $D / M / 1$ queue with a finite workload capacity is same as that of the residual workload in an $M / D / 1$ queue with same bound on the workload capacity.

This phenomenon can be viewed as a duality between the time averages in an $M / D / 1$ queue and the customer averages in a $\mathrm{D} / \mathrm{M} / 1$ system.

\section{$6 \quad$ Linear Loss Rate: $\lambda(x)=\lambda x$}

In this section we give a general expression for the stationary distribution of the window size process with a linear increase profile under a linear loss rate assumption for general window decrease profile. We then provide the stationary distribution for Scalable TCP and HighSpeed TCP under linearly increasing loss rates. This case is of practical interest since a linear loss rate is seen by the connection when each packet is dropped with a fixed probability $p$ (see [15]).

\subsection{Additive Increase General Decrease AWP}

We now consider an AWP with a linear increase profile and assume that the loss rate is linearly increasing with the window size, i.e., $\lambda(u)=\lambda u$ for some $\lambda>0$. This is the case of practical interest because the standard congestion avoidance phase of TCP (as proposed in [7]) is linearly increasing. Moreover, recently proposed HighSpeed TCP [14] opens up

$\mathrm{RR} \mathrm{n}^{\circ} 5262$ 
a possibility of wide range of protocols where the window increase is approximately linear (with a larger additive increase constant as compared to the standard TCP) and the decrease is given by some window dependent factor. As mentioned already, loss rates in cases where each packet is dropped with a fixed probability and TCP drops its window at most once in a round-trip time indeed increase linearly with the window size of the AWP. In the following we assume that the increase profile is same as that in standard TCP, i.e., window increases by one unit per unit time; this can be assumed because an increase profile with a different (constant) slope can be mapped to that of unit slope while keeping the loss rate linear using the transformation introduced in Section 2.1.

Proposition 3 For $x$ such that $G^{1}<x<G^{0}$, the stationary distribution is

$$
\pi(x)=c_{1} e^{\frac{-\lambda x^{2}}{2}}
$$

for $c_{1}=\lambda G^{0}\left[1-\Pi\left(G^{0}\right)\right] e^{\frac{\lambda G^{0}}{2}}$. For $x \in\left(G^{l}, G^{l-1}\right), m \geq l>1$,

$\pi(x) e^{\lambda \frac{x^{2}}{2}}=\sum_{j=0}^{l-1} c_{l-j} \lambda^{j} \int_{u_{1}=H(x)} \ldots \int_{u_{j}=H\left(u_{j-1}\right)} u_{1} e^{\lambda \frac{G\left(u_{1}\right)^{2}-u_{1}{ }^{2}}{2}} \ldots u_{j} e^{\lambda \frac{G\left(u_{j}\right)^{2}-u_{j}{ }^{2}}{2}} d u_{j} \ldots d u_{1}$,

where $c_{j}$ are some constants to be computed using the exact form of $G(\cdot)$.

Proof: See Appendix E.

For numerical computations, we can use continuity of $\Pi(\cdot)$ at the boundaries $G^{i}$ to compute $c_{j}$ 's like done in Section 5.1. Now we work out the above expression for the case of AIMD protocol.

\subsubsection{The Case of Standard TCP: AIMD}

For the case of standard TCP with linear window dependent loss rate, [15] has obtained an expression for the stationary window size distribution. Their method however requires guessing the expression for the stationary distribution and then proving it inductively. Our approach is to directly determine the distribution without need for guessing. This is a considerable amount of simplification for the case of a general AWP as we will see in section 6.2 that the distribution can in general be not straightforward to guess. We will also see in the present section that the very nature of AIMD makes it (relatively) easier to predict the structure of the stationary distribution.

Proposition 4 For $x \in\left[G^{l}, G^{l-1}\right]$,

$$
\pi(x)=\sum_{j=0}^{l-1} c_{l-j} b_{j} e^{a_{j} x^{2}}
$$

where $b_{j}=\Pi_{n=1}^{j} \frac{\lambda}{2 K \sum_{\kappa=0}^{n} \beta^{-2 \kappa}}, K=\lambda \frac{\beta^{2}-1}{2}$ and $a_{j}=\left(\frac{K}{\beta^{2}}\left(\sum_{\kappa=0}^{j-1} \beta^{-2 \kappa}\right)-0.5 \lambda\right)$, with $b_{0}=1$. Here $c_{l}$ are integration constants. 
Proof See Appendix $\mathrm{F}$ for proof and closed form expression for $c_{j} \mathrm{~s}$ in terms of Gamma functions.

A similar expression has been obtained in [15]. However, [15] provides only the recursion for the integration constants appearing in their expressions. They need to compute the value of these constants using numerical integration at the end, whereas we have a closed form expression for these constants. The model of [15] allows for the window size of 0 packets (during time-out periods) and also allows multiplicative decrease while window size is less than one packet, this makes their recursion of infinite length. This also results in a large discrepancy in the distributions for small window sizes. As we pointed out in Section 3.1, allowing for a window size of less than one packet may result in a model that is stable in only a restricted set of parameter values. Further, [15] also accounts for timeout periods and also distinguishes between triple dupack losses and timeout based loss recovery. It is seen that our model is easily extended to consider these possibilities (though we believe that these phenomenon are rare, hence not of significant importance, when the TCP-SACK version is used).

\subsection{MIMD Protocols with Linear Loss Rates}

For the case of MIMD protocols, the window evolution is described as follows. In case of no loss in interval $[t, t+\Delta]$, the window increases to (assuming no upper bound on window size)

$$
x_{t+\Delta}=x_{t}+\alpha x_{t} \Delta+o(\Delta),
$$

for some $\alpha>0$. In case of a loss in interval $[t, t+\Delta]$, the window decreases to

$$
x_{t+\Delta}=\left(\beta x_{t}\right) \vee 1+o(\Delta)
$$

where $1>\beta>0$ is the multiplicative decrease constant. The natural lower bound of $x_{t} \geq 1$ packet applies.

The window is bounded below by a constraint of $x_{\min }$ packet. The window evolution under such scenario is depicted in Figure 4. The figure shows that the window starts evolving from an initial value of 1 packet. There are some multiplicative decrease of window owing to random losses. The vertical axis is shown to be divided into various intervals

$I_{k} \triangleq\left(\beta^{-k}, \beta^{-k-1}\right]$. Here $\beta<1$ is the multiplicative decrease factor. The significance of these regions is that if a loss event occurs when the window size is in interval $I_{k+1}$ then the reduced window is in region $I_{k}$. We remark here that we are not working with the transformed window having a linear increase profile as introduced in Section 2.1. The upper bound on $x$ is $M=\beta^{-m}$ for some $m$.

For this case the following Kolmogorov equations can be obtained for $x<\beta^{-m+1}$,

$$
\pi(x) \alpha x=\int_{u=x}^{u=\frac{x}{\beta}} \pi(u) \lambda u d u,
$$

$\mathrm{RR} \mathrm{n}^{\circ} 5262$ 


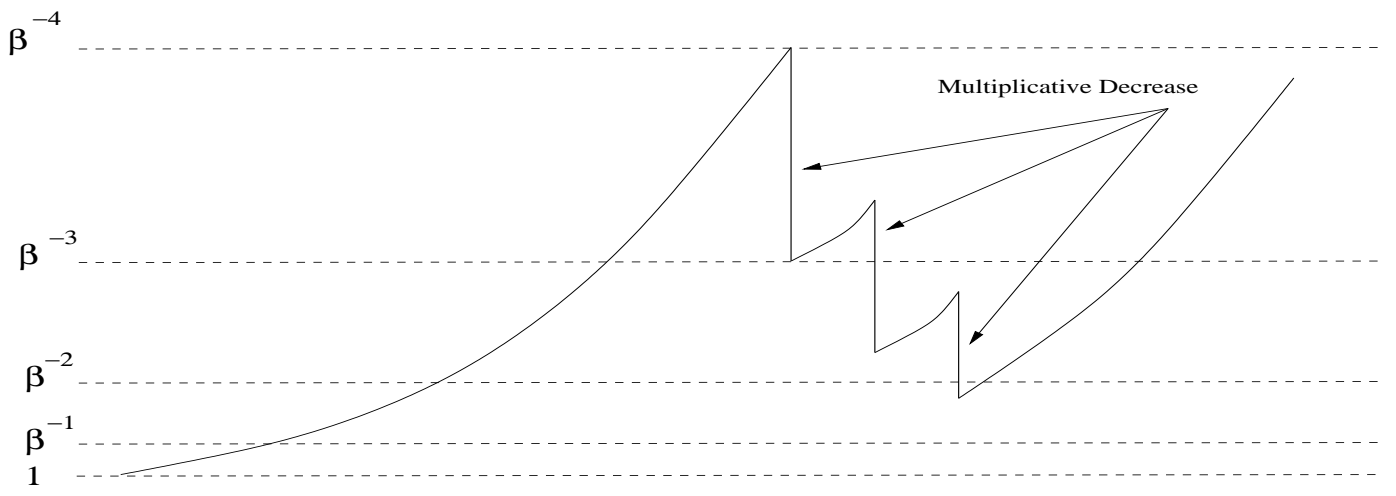

Figure 4: Window evolution under MIMD protocol like Scalable TCP with a lower bound on window size.

where $\alpha$ is as in Equation 9. Denote now, by an abuse of notation, $\lambda=\frac{\lambda}{\alpha}$. The above Kolmogorov equation is then

$$
\pi(x) x=\int_{u=x}^{u=\frac{x}{\beta}} \pi(u) \lambda u d u .
$$

Proposition 5 The steady state probability density function of the window size under linear loss rate is given by, if $x \in I_{m-k}, k \geq 2$,

$$
\pi(x)=M P_{M} \sum_{j=1}^{k} c_{i}^{(k)} \frac{\lambda}{x \beta^{i-1}} e^{\frac{\lambda x}{\beta^{i-1}}} .
$$

Here $c_{i}^{(k)}$ are some constants obtained by normalising $\pi(\cdot)$ to get a probability measure and $P_{M}$ is the probability mass at $M$.

Proof: See Appendix G for expressions for $P_{M}$ and $c_{i}^{(k)}$.

One is often interested in finding the moments of the window process. This can be obtained easily without need to compute the coefficients $c_{i}^{(k)}$ as follows. We assume here that $x_{\text {min }}=0$ and $M=\infty$; this is expected to approximate the case when the upper and lower bounds are not attained frequently. The Kolmogorov equation obtained above is multiplied by $x^{j-1}, j \geq 0$ to obtain

$$
\begin{aligned}
\pi(x) x^{j} & =x^{j-1} \int_{u=x}^{u=\frac{x}{\beta}} \pi(u) \lambda u d u \\
\Rightarrow \int_{x=0}^{\infty} \pi(x) x^{j} d x & =\int_{x=0}^{\infty} x^{j-1} \int_{u=x}^{u=\frac{x}{\beta}} \pi(u) \lambda u d u d x
\end{aligned}
$$

INRIA 


$$
\begin{aligned}
E[X]^{j} & =\int_{u=0}^{\infty} \int_{x=\beta u}^{u} x^{j-1} d x \pi(u) \lambda u d u \\
\Rightarrow E[X] & =\frac{1}{-\lambda \ln (\beta)} \\
E[X]^{j+1} & =\frac{j}{\lambda\left(1-\beta^{j}\right)} E[X]^{j}=\frac{j !}{\lambda^{j} \Pi_{i=1}^{j}\left(1-\beta^{i}\right)} E[X], j \geq 1,
\end{aligned}
$$

thus we get all the moments of the window size distribution. We see from the above that the tail of the window size distribution is exponentially decaying and that all the moments exist.

\subsection{HighSpeed TCP}

HighSpeed TCP (HSTCP, [14]) updates the window in a round-trip time according to the following rules: In case of no loss in a round-trip time during which the window size was $w$, the window is incremented by a window dependent quantity, denoted $a(w)$, so that the new window size is $w+a(w)$, and in case of a packet drop on a round-trip time, the window is decremented by a window dependent factor $b(w)$ so that the new window size is $(1-b(w)) w$. The window size is bounded by two values $w_{l}$ and $w_{h}$ and $a(w)=\frac{2 w^{2} b(w) p(w)}{2-b(w)}, b(w)=$ $\frac{\log \left(\frac{w}{w_{l}}\right)}{\log \left(\frac{w_{h}}{w_{l}}\right)}\left(b_{h}-b_{l}\right)+b_{l}, \quad p(w)=\exp \left(\frac{\log \left(\frac{w}{w_{l}}\right)}{\log \left(\frac{w_{h}}{w_{l}}\right)} \log \left(\frac{p_{h}}{p_{l}}\right)+\log \left(p_{l}\right)\right)$, where $b_{h}=b\left(w_{h}\right), b_{l}=$ $b\left(w_{l}\right), p_{l}=p\left(w_{l}\right)$ and $p_{h}=p\left(w_{h}\right)$ are design parameters. It is suggested in [14] to set $w_{l}=31$ and $p_{l}=\frac{1.5}{w_{l}^{2}}$. Note that $p(w)=\nu w^{\mu}$ where $\mu=\frac{\log \left(\frac{p_{h}}{p_{l}}\right)}{\log \left(\frac{w_{h}}{w_{l}}\right)}$ and $\nu=\frac{p_{l}}{w_{l}^{\mu}}$ and $b(w)=A \log (w)+B$ with $A=\frac{b_{h}-b_{l}}{\log \left(\frac{w_{h}}{w_{l}}\right)}$ and $B=b_{l}-A \log \left(w_{l}\right)$. Since $b_{h}<b_{l}, A<0$ and since $w_{h} \geq w_{l}, p_{h} \leq p_{l} \Rightarrow \mu<0$. We observe that, if $R$ represents the round-trip time, then $w(t+R)=w(t)+a(w(t))=w(t)+\frac{2 w^{2}+\mu b(w) \nu}{2-b(w)}$. This equation shows the importance of parameter $\mu$ in understanding the behavior of HSTCP. For example, $\mu=-2$ implies that HSTCP is similar to the standard AIMD algorithm of TCP where in each round-trip time, the window is incremented by a small value (in this case $\frac{2 b(w) \nu}{2-b(w)} \approx \nu b(w)$ ). If we take $\mu>-2$, then we get a protocol whose window increment increases with the window, for example, taking $\mu=-1$ implies that HSTCP is similar to Scalable TCP in behavior since now the increment is approximately linear in window size. This observation suggests need for care in tuning the HSTCP parameters. It also implies the possibility of existence of a choice of $\mu \in(-2,-1)$ which is neither as aggressive as Scalable TCP nor conservative as standard TCP. Now we analyse HSTCP assuming that $A \approx 0$ so that the decrease factor is constant. Since the form of function $b(w)$ is a design choice (see [14]), this form of $b(w)$ can be chosen for simplicity of implementation. Further, for this choice of $b(w)$ we can find the stationary window size distribution for the protocol for different values of $\mu$ as follows: First observe that for $b(w)=B$, the increase profile of the protocol is $f(w)=\frac{2 \nu B w^{2+\mu}}{2-B}$ and assuming a linear loss rate $\lambda(w)=\lambda w$, the Kolmogorov equation can be transformed to the case of unit loss rate as in Section 3 to get, $\frac{2 \nu B w^{1+\mu}}{\lambda(2-B)} \pi(w)=\int_{u=w}^{\frac{w}{1-B}} \pi(u) d u$. Now, this 
Kolmogorov equation, when transformed to the case of AWP with linear increase profile, becomes $\tilde{\pi}(y)=\int_{u=y}^{\frac{y}{(1-B)^{-\mu}}} \tilde{\pi}(u) d u$. The closed form solution for this equation is known from [5] as this corresponds to the case of AIMD protocol with constant loss rate (here we have used the fact that $-\mu>0$ so that $\left.(1-B)^{-\mu}<1\right)$.

\section{Incorporating Multiple Reductions and General De- crease}

The analysis of Section 6.1 and 6.2 assumed that a loss instant results in a single (deterministic) window reduction. This is frequently the case as the new versions of TCP are required not to undergo multiple window reductions in a single round-trip time. However, there can be possibility, owing to large number of losses, that the TCP sender undergoes multiple window reductions in a single round-trip time. Typically, the number of such reductions in a single round-trip time will be random. We consider this situation in the current section. In what follows, we allow for the loss rate to be dependent on the current window and make no explicit structural assumption for this dependence.

To model the (random) number of window reductions, we assume that the loss process is batch Poisson of rate $\lambda(u)$ and given a loss event, the batch size (the number of associated reductions) is at least $k$ with probability $p_{k}$. Note that $p_{1}=1$. Let $\lambda(u) p_{k} \triangleq q_{k}(u)$. The Kolmogorov equations now are, for $y \in\left(G^{l}, G^{l-1}\right)$ :

$$
\Pi^{\prime}(y)=\sum_{k=0}^{l-1} \int_{u=H^{k}(y)}^{H^{k+1}(y)} q_{k+1}(u) d \Pi(u)
$$

Let $q_{k, l} \triangleq \sup _{u \in\left(G^{l}, G^{l-1}\right)} q_{k}(u)$. We see that

$$
\begin{aligned}
\pi(y) & =\sum_{k=0}^{l-1} \int_{u=H^{k}(y)}^{H^{k+1}(y)} q_{k+1}(u) \pi(u) d u \\
& =\sum_{k=0}^{l-1} \int_{u=H^{k}(y)}^{G^{l-k-1}} q_{k+1}(u) \pi(u) d u+\sum_{k=0}^{l-1} \int_{u=G^{l-k-1}}^{H^{k+1}(y)} q_{k+1}(u) \pi(u) d u \\
& \leq \int_{u=y}^{G^{l-1}} q_{1}(u) \pi(u) d u+\sum_{k=1}^{l-1} \int_{u=H^{k}(y)}^{G^{l-k-1}} q_{k+1, l-k} \pi(u) d u+\sum_{k=0}^{l-1} \int_{u=G^{l-k-1}}^{H^{k+1}(y)} q_{k+1, l-k-1} \pi(u) d u \\
& \leq \int_{u=y}^{G^{l-1}} q_{1}(u) \pi(u) d u+\sum_{k=1}^{l-1} \int_{u=G^{l-k}}^{G^{l-k-1}} q_{k+1, l-k} \pi(u) d u+\sum_{k=0}^{l-1} \int_{u=G^{l-k-1}}^{G^{l-k-2}} q_{k+1, l-k-1} \pi(u) d u \\
& =\int_{u=y}^{G^{l-1}} q_{1}(u) \pi(u) d u+\sum_{k=1}^{l-1} \int_{u=G^{l-k}}^{G^{l-k-1}} q_{k+1, l-k} \pi(u) d u+\sum_{k=0}^{G^{l-k-2}} \int_{u=G^{l-k-1}} q_{k+1, l-k-1} \pi(u) d u
\end{aligned}
$$

INRIA 


$$
\leq \int_{u=y}^{G^{l-1}} \tilde{q}_{1}(y) \pi(u) d u+\theta_{l}
$$

where $\theta_{l}$ is an upper bound on the remaining term above and $\tilde{q}_{1}(y) \triangleq \sup _{y \leq u \leq G^{l-1}} q_{1}(u)(\leq$ $\left.q_{1, l}\right)$. It follows that

$$
\pi(y) \leq \theta_{l} e^{\tilde{q}_{1}(y)\left(G^{l-1}-y\right)}
$$

Here $\theta_{l}$ can be found using a recursive equation as follows.

$$
\begin{aligned}
\theta_{l}= & \sum_{k=1}^{l-1} \int_{u=G^{l-k}}^{G^{l-k-1}} q_{k+1, l-k} \theta_{l-k} e^{\lambda\left(G^{l-k-1}-G^{l-k}\right)} d u \\
& +\sum_{k=0}^{l-2} \int_{u=G^{l-k-1}}^{G^{l-k-2}} q_{k+1, l-k-1} \theta_{l-k-1} e^{\lambda\left(G^{l-k-2}-G^{l-k-1}\right)} d u+\int_{u=G^{0}}^{\infty} q_{l, 0} \pi(u) d u .
\end{aligned}
$$

This can be easily computed recursively, given $\theta_{1}$ and $\pi(u), u>G^{0}$. Exact value of $\theta_{1}$ and $\pi(u), u>G^{0}$ is computed below.

Remark: We can also use a relatively loose bound to get a simpler representation of $\theta_{l}$ as follows. Note that $q_{k+1, j} \leq q_{k, j}$ for all values of $j$ and $k$. Thus,

$$
\begin{aligned}
\theta_{l}= & \sum_{k=1}^{l-1} \int_{u=G^{l-k}}^{G^{l-k-1}} q_{k+1, l-k} \theta_{l-k} e^{\lambda\left(G^{l-k-1}-G^{l-k}\right)} d u \\
& +\sum_{k=0}^{l-2} \int_{u=G^{l-k-1}}^{G^{l-k-2}} q_{k+1, l-k-1} \theta_{l-k-1} e^{\lambda\left(G^{l-k-2}-G^{l-k-1}\right)} d u+\int_{u=G^{0}}^{\infty} q_{l, 0} \pi(u) d u \\
\leq & \sum_{k=1}^{l-1} \int_{u=G^{l-k}}^{G^{l-k-1}} q_{k, l-k} \theta_{l-k} e^{\lambda\left(G^{l-k-1}-G^{l-k}\right)} d u \\
& +\sum_{k=0}^{l-2} \int_{u=G^{l-k-1}}^{G^{l-k-2}} q_{k+1, l-k-1} \theta_{l-k-1} e^{\lambda\left(G^{l-k-2}-G^{l-k-1}\right)} d u+\int_{u=G^{0}}^{\infty} q_{l, 0} \pi(u) d u \\
= & \sum_{k=0}^{l-2} \int_{u=G^{l-k-1}}^{G^{l-k-2}} q_{k+1, l-k-1} \theta_{l-k-1} e^{\lambda\left(G^{l-k-2}-G^{l-k-1}\right)} d u \\
& +\sum_{k=0}^{l-2} \int_{u=G^{l-k-1}}^{G^{l-k-2}} q_{k+1, l-k-1} \theta_{l-k-1} e^{\lambda\left(G^{l-k-2}-G^{l-k-1}\right)} d u+\int_{u=G^{0}}^{\infty} q_{l, 0} \pi(u) d u \\
= & 2 \sum_{k=0}^{l-2} \int_{u=G^{l-k-1}}^{G^{l-k-2}} q_{k+1, l-k-1} \theta_{l-k-1} e^{\lambda\left(G^{l-k-2}-G^{l-k-1}\right)} d u \\
& +\int_{u=G^{0}}^{\infty} q_{l, 0} \pi(u) d u .
\end{aligned}
$$

$\mathrm{RR} \mathrm{n}^{\circ} 5262$ 
Exact value of $\theta_{1}$ and $\pi(u), u>G^{0}$

We know, however that for $y>G^{1}$, we get the simple equation (noting that $q_{1}=\lambda$ ):

$$
\begin{aligned}
\Pi^{\prime}(y) & =\lambda \int_{u=y+}^{\infty} \pi(u) d u \\
\Pi^{\prime}(y) & =\lambda(1-\Pi(y)) \\
\frac{d}{d y} \Pi(y) e^{\lambda y} & =\lambda e^{\lambda y} \\
\Pi(y) e^{\lambda y} & =\int_{u=0}^{y} \lambda e^{\lambda u} d u+\Pi\left(G^{1}\right) \\
& =e^{\lambda y}-1+\Pi\left(G^{1}\right) \\
\Pi(y) & =1-e^{-\lambda y}\left(1+\Pi\left(G^{1}\right)\right) \\
\pi(y) & =\lambda e^{-\lambda y}\left(1+\Pi\left(G^{1}\right)\right)
\end{aligned}
$$

Comparing this with Equation 10, we get $\theta_{1}$ in terms of $\Pi\left(G^{1}\right)$. Integrating Equation 10, and setting the integral to unity, we get a lower bound on $\Pi\left(G^{1}\right)=\Pi(G(M))$.

If we assume that the loss rate is independent of window size, we get:

$$
\pi(y)=\sum_{k=0}^{l-1} \int_{u=H^{k}(y)}^{G^{l-k-1}} q_{k+1} \pi(u) d u+\sum_{k=0}^{l-1} \int_{u=G^{l-k-1}}^{H^{k+1}(y)} q_{k+1} \pi(u) d u
$$

\section{$8 \quad$ Stability Results}

An important problem now is to study the stability of the process $\left\{x_{t}\right\}$ for a given AQM or loss profile $(\lambda(\cdot))$ and a given AWP increase/decrease profile (the functions $f(\cdot)$ and $g(\cdot))$. Alternatively, for a given AWP, one would like to design an AQM profile; this design process must obviously address the issue of the stability of the window process under the chosen AQM profile. By stability here we mean that the window size (or the buffer occupancy) should, with large probability, take values in compact sets. In the following we give necessary and sufficient conditions for stability of the $\left\{x_{t}\right\}$ process; these condition can then be used in the design of AQM profile.

In this section we are interested in study of the stability of the $\left\{x_{t}\right\}$ process (which has been observed to be a special instance of PDMPs in Section 2.5). Available study in literature on stability of PDMPs (see for example [19]) shows that the process $\left\{x_{t}\right\}$ is stable iff the Markov chain $\left\{x_{n}\right\}$ embedded at the end of jump instants is stable. [19] also gives drift criteria type condition for the stability of the embedded chain $\left\{x_{n}\right\}$. Using this approach one requires to construct a Lyapunov function every time there is a slight modification in the jump or deterministic evolution phase of the PDMP. This gives only sufficient condition for stability. 
We first provide a stochastic ordering result which says that the steady-state window process with a larger upper bound is stochastically larger than the process with a smaller upper bound. We then give a necessary and sufficient condition for existence and uniqueness of an invariant measure for the window process such that this measure has most of its mass concentrated on compact sets. We then provide a transformation from a process with statedependent loss rate to one with state-independent loss rate. The necessary and sufficient stability condition for state-independent loss rate is then seen to apply to a general AWP with a general loss rate. We also give a simpler necessary condition for stability of an AWP under a general loss rate $\lambda(\cdot)$. Since the loss rate $\lambda(\cdot)$ can take very different forms if an Active Queue Management (AQM) scheme is used, the study of this section also applies to the interaction of an AQM and AWP. Note here that using a drift criteria based approach would require to change the Lyapunov function every time either AWP or AQM is changed. Our method however gives a condition for a general AWP and AQM. Moreover, the analysis presented in the paper till now to obtain stationary distribution for the bounded window process is useful in the criteria obtained in this section.

\subsection{Construction of Bounded Processes}

Throughout in this section we will assume that the deterministic increase of the $\left\{x_{t}\right\}$ process is linear. We have already seen that an AWP with a general increase profile can be continuously transformed to the one with linear increase. The assumption on $\left\{x_{t}\right\}$ process made above is then justified by the fact that a continuous transformation preserves compactness of sets and hence will also preserve stability property.

Consider the sequence $\left\{x_{t}^{M}\right\}$ of PDMPs bounded above by a constant $M$. Between jumps, the process $\left\{x_{t}^{M}\right\}$ increases linearly. However, if the process achieves the level $M$, it stays there until next jump (which occurs at rate $\lambda(M)$ ). For each such $M$, let $\pi_{M}(\cdot)$ and $\Pi_{M}(\cdot)$ denote, respectively, the stationary density and distribution for the bounded PDMP $\left\{x_{t}^{M}\right\}$ (we assume existence of these). Let $P_{M}$ denote the point mass at $M$ of the stationary probability for $\left\{x_{t}^{M}\right\}$. The steady state Kolmogorov equation satisfied by $\pi_{M}(\cdot)$ is (recall the function $B(\cdot, \cdot)$ from Section 2.5)

$$
\pi_{M}(y)=P_{M} \lambda(M) B(M, y)+\int_{x=y}^{M-} \lambda(x) B(x, y) d \Pi_{M}(x)
$$

Remark: The existence of such $\Pi_{M}(\cdot)$ follows from the existence of solution for the Fredholm equations of second type.

\subsection{Limit of the Bounded Processes}

It is to be noted here that the convergence of the process $\left\{x_{t}^{M}\right\}$ to $\left\{x_{t}\right\}$ as $M \rightarrow \infty$ follows from arguments similar to those in [18]. Now, if a stationary distribution $\Pi(\cdot)$ exists for the 
original PDMP $\left\{x_{t}\right\}$, it must satisfy the steady state Kolmogorov equation

$$
\pi(y)=\int_{x=y}^{\infty} \lambda(x) B(x, y) d \Pi(x)
$$

Proposition 6 The process $\left\{x_{t}\right\}$ is stable and has an invariant measure $\pi(\cdot)$ if $\Pi_{M}(\cdot)$ forms a tight family of probability measures.

Proof: See Appendix H. (See [20] for definition of tight family of probability measures.) •

Above we showed, via a weakly convergent subsequence, that an invariant probability measure exists if the sequence $\left\{\Pi_{M}(\cdot)\right\}$ is tight. However, it may be possible that there are many subsequences of $\left\{\Pi_{M}(\cdot)\right\}$ converging to different weak limits. In that case each of these weak limits is an invariant measure. Below we give sufficient conditions under which such a situation does not arise. This condition is satisfied by AWP controlled window evolution with state-independent loss rate.

Lemma 1 If $B(\cdot, y)$ is a unit step function that is strictly decreasing for each $y$ and if $\lambda(x)$ is independent of state $x$ (but may depend on time), then the sequence $\left\{\Pi_{M}(x)\right\}$ is monotone non-increasing in $M$ for each fixed $x$, i.e., $x^{M_{1}} \leq_{s t} x^{M_{2}}$ for $M_{1} \leq M_{2}$.

Proof: Consider two bounds $M$ and $M+1$. Let the corresponding bounded processes be denoted $Y^{1}(t)$ and $Y^{2}(t)$. Fix initial points $y^{1}$ and $y^{2}>y^{1}$ for these processes. Fix also a particular sample path $\left\{T^{n}\right\}$ of loss instants over time for both the processes $Y^{1}(t)$ and $Y^{2}(t)$. Because of our assumption of state independent loss rate this sample path has a probability independent of the two processes. This also is the only place where we have used the assumption of state independent loss rate $\lambda(\cdot)$.

Consider $n^{\text {th }}$ loss instant $T^{n}$. Assume that $Y^{1}\left(T^{n}\right) \leq Y^{2}\left(T^{n}\right)$. We now find condition under which $Y^{1}\left(T^{n+1}\right) \leq Y^{2}\left(T^{n+1}\right)$. This will then imply that $Y^{1} \stackrel{<}{\text { st }} Y^{2}\left(Y^{1}\right.$ is stochastically less than $Y^{2}$ ) thus implying monotonicity of $\Pi_{M}(y)$ over $M$. Since the increase in the two processes is linear (or, at most, clipped at a level) in the interval $\left[T^{n}+, T^{n+1}\right]$, it follows that $Y^{1}(t) \leq Y^{2}(t), \forall t \in\left[T^{n}+, T^{n+1}\right]$. Now, if the jumps are deterministic, i.e., the distribution $B(x, \cdot)$ is a shifted unit-step function for each $x$, and if $B(x, y)$ is decreasing in $x$ for each fixed $y$ then it is easily seen that $Y^{1}\left(T^{n+1}\right) \leq Y^{2}\left(T^{n+1}\right)$. Thus it follows that $Y^{1}(t) \leq Y^{2}(t)$ for all $t \geq 0$ if $Y^{1}(0) \leq Y^{2}(0)$. Thus, it follows that $Y^{1} \stackrel{\leq}{\text { st }} Y^{2}$.

The above proof shows that if $Y^{1}(0) \leq Y^{2}(0)$ then $Y^{1}(t) \leq Y^{2}(t), \forall t$. However it is easy to see that if $B(x, y)$ is unit-step function and is strictly decreasing ${ }^{3}$ in $x$ for each fixed $y$ then $Y^{1}(0)>Y^{2}(0)$ implies that $Y^{1}(t) \leq Y^{2}(t)$ eventually. This is because in this case the above arguments imply that the difference $Y^{1}\left(T^{n}\right)>Y^{2}\left(T^{n}\right)$ then $Y^{1}\left(T^{n}\right)-Y^{2}\left(T^{n}\right)>$ $Y^{1}\left(T^{n+1}\right)-Y^{2}\left(T^{n+1}\right)$ so that $Y^{2}\left(T^{n}\right)$ will approach $Y^{1}\left(T^{n}\right)$ and then the above arguments imply that $Y^{2}(t)$ will exceed $Y^{1}(t)$.

Remark The monotonicity property obtained above is not an intuitive result. This is because for the bounded processes, though the solution to the Kolmogorov equations can

\footnotetext{
${ }^{3}$ by this we mean that for $x_{1}>x_{2}, \exists y: B\left(x_{1}, y\right)<B\left(x_{2}, y\right)$
} 
be monotone, the normalization required to make them probability measures can have an unpredictable effect in general. In our case, however, it turns out that monotonicity is preserved by the required normalisation.

Remark Since the proof of Lemma 1 is sample-path wise and does not use the exact form of the increase profile, we see that it applies also to the system with a general increase profile and a constant loss rate.

We have shown above that the sequence $\left\{\Pi_{M}(x)\right\}$ is monotone non-increasing for specific structure of $B(\cdot, \cdot)$. Monotonicity of $\left\{\Pi_{M}(x)\right\}$ for each $x$ implies that there is a unique pointwise limit of the sequence $\left\{\Pi_{M}(x)\right\}$ for each $x$. This remains valid whether or not $\left\{\Pi_{M}(\cdot)\right\}$ is tight. It is now easy to see that if $\left\{\Pi_{M}(\cdot)\right\}$ is tight then there exists a unique weak limit of $\left\{\Pi_{M}(\cdot)\right\}$. If, however, $\left\{\Pi_{M}(\cdot)\right\}$ is not tight then it follows that there exists a value $0<r<1$ such that $\lim _{M \rightarrow \infty} \Pi_{M}(x)>r$ for all $x$. We have thus

Proposition 7 Under the conditions of Lemma 1, the process $\left\{x_{t}\right\}$ is stable and has a unique invariant measure $\pi(\cdot)$ iff $\Pi_{M}(\cdot)$ forms a tight family of probability measures.

Now we state an important result which will be used in study of stability of an AWP under a general loss rate.

Theorem 6 The process $\left\{x_{t}\right\}$ is unstable iff $\lim _{M \rightarrow \infty} P_{M}>0$.

Proof: Since if $\lim _{M \rightarrow \infty} P_{M}>0$, the sequence of probability measures $\Pi_{M}(\cdot)$ can not be tight, the proof follows from Proposition 7.

If the sequence $\Pi_{M}(\cdot)$ is tight then it is easily seen, using monotonicity of $\Pi_{M}(\cdot)$, that $\lim _{M \rightarrow \infty} P_{M}=0$. Thus the reverse implication also follows.

Remark Results relating stability and tightness of probability measures are known in context of Markov chains also (see [29]). The results of this section are for Markov processes of a specific kind and the criteria for checking the tightness as in Theorem 6 , obtained from establishing monotonicity probability measure over constrained state spaces is new. It needs to be emphasized that the above result may not be true in context of a general PDMP, because for proof of the Theorem 6 we have used the property of an AWP that the point to which an AWP jumps is a nondecreasing function of the window size.

We now make the following conjecture,

Conjecture 1 For the case of state dependent loss rate $\lambda(\cdot)$, the if part of Theorem 6 remains valid, i.e., if $\lim _{M \rightarrow \infty} P_{M}>0$ then the process $\left\{x_{t}\right\}$ is unstable.

\subsection{Application of the Stability Result}

We now establish a necessary and sufficient criteria for stability of a general AWP (general increase and decrease profile) under a general loss rate. Using a transformation introduced in Section 4 brings us in the framework of Theorem 6 which assumes that the loss rate is constant and the AWP has a linear increase profile. Thus, without loss of generality, we can assume that the protocol under consideration has a linear increase profile and the loss rate

$\mathrm{RR} \mathrm{n}^{\circ} 5262$ 
is unity. It is clear that the original system is stable if this transformed system is stable. Now we use Theorem 4 that provides an expression for the probability mass at the bound $M$ for the bounded process with linear increase and unit loss rate and obtain (recall the notation of Theorem 4)

Theorem 7 A general AWP controlled window evolution is stable under a general loss rate iff

$$
e^{-M}\left[\sum_{j_{1}=1}^{m-1} \sum_{j_{2}=1}^{j_{1}-1} \ldots \sum_{j_{m-2}=1}^{j_{m-3}-1} q_{m, j_{1}} q_{j_{1}, j_{2}} \ldots q_{j_{m-2}, 1}\right]^{-1} \rightarrow 0 \text { as } m \rightarrow \infty .
$$

Proof Follows from Theorem 4 since we know that the AWP is stable if and only if $P_{M} \rightarrow 0$ as $M \rightarrow \infty$ from Theorem 6 .

Remark Recall that Lemma 1 applies also to the system with a general increase profile and constant loss rate. Since, for the window evolution under a general increase profile $f(\cdot)$ and a general loss rate $\lambda(\cdot)$, its stationary probability measure $\pi(\cdot)$ is such that $\frac{\pi(x) \lambda(x)}{E[\lambda(X)]}$ satisfies the Kolmogorov equation for a system with increase profile $\frac{f(x)}{\lambda(x)}$ (see Section 4), the function $\Pi_{M}(x)=\int_{u=x_{\text {min }}}^{x} \frac{\pi(u) \lambda(u)}{E[\lambda(X)]} d u$ is monotone in $M$ for each $x$. This result carries over to the corresponding queueing system with finite workload capacity in a natural way.

We now establish stability conditions for the MIMD algorithm (Scalable TCP and Slow Start) with a constant state-independent loss rate. We then give a stability criteria for the case of general AWP with a constant loss rate and then we provide an instability criteria for the case of general AWP with a general loss rate.

Theorem 8 For an AWP with a linear increase profile and a constant loss rate, a necessary condition for stability is that

$$
1-e^{-\lambda M}\left[\sum_{n=1}^{m}\left[\Pi_{j=1}^{n}\left(1-e^{-\lambda l_{j}}\right)\right] e^{-\lambda G^{n}}+e^{-\lambda M}\right]^{-1}
$$

converge to a positive value as $M \rightarrow \infty$. Here $l_{j}=G^{j-1}-G^{j}$.

Proof See Appendix I.

Now, for the Additive increase constant decrease case (which we get when transforming a MIMD protocol to the linear increase protocol, which is the case of scalable TCP), we denote the constant decrease by $\theta$. Application of the above result leads us to the following

Theorem 9 A necessary condition for stability of additive increase constant decrease algorithms with a constant drop rate $\lambda$ used by the $A Q M$ is that

$$
\lambda>\frac{\ln 2}{\theta} .
$$


Proof: Noting that $\theta_{1}=(1-\Pi(M)) e^{-\lambda M}$, we see that $G^{n}=M-n \theta$ and $m=\frac{M}{\theta}$,

$$
(1-\Pi(M)) \geq \frac{1}{1+\sum_{n=1}^{\frac{M}{\theta}}\left(e^{\lambda \theta}-1\right)^{n}}
$$

so, if $1>e^{\lambda \theta}-1$, or $e^{\lambda \theta}<2$, we see that the sum in the denominator above converges to a finite strictly positive constant, say $r$ thus we get that for any large $M, 1-\Pi(M) \geq r>0$. Since $r$ is independent of $M$, we see that under the condition $e^{\lambda \theta}<2$, the unbounded window evolution can not be stable, i.e., $e^{\lambda \theta}>2$ or $\lambda>\frac{\ln 2}{\theta}$ is a necessary condition for stability.

Now we apply the above method to the case where the drop rate is state dependent. We are assuming here that Theorem 6 is applicable to this case as well (Conjecture 1).

Theorem 10 For an AWP with a linear increase profile and a general loss rate, a sufficient condition for instability is that

$$
\left[\frac{\kappa_{1}}{\lambda(M)} e^{-\lambda(M) M}+\sum_{j=1}^{m} \frac{\kappa_{j}}{\lambda_{j}}\left(e^{-\lambda_{j} G^{j}}-e^{-\lambda_{j} G^{j-1}}\right)\right]^{-1}
$$

converges to a positive values as $M \rightarrow \infty$. Here $l_{j}=G^{j-1}-G^{j}, \kappa_{j}=\frac{\theta_{j}}{1-\Pi(M)}, \lambda_{i}=$ $\sup _{G^{i-1} \geq y \geq G^{i}} \lambda(y), \theta_{i}^{\prime}=\int_{u=G^{i-1}}^{G^{i-2}} \lambda(u) \theta_{i-1} e^{-\lambda_{i-1} u} d u, \theta_{i}=\theta_{i}^{\prime} e^{\lambda_{i} G^{i-1}}$ and $\theta_{1}^{\prime}=\lambda(M)(1-$ $\Pi(M))$.

Proof See Appendix J.

Since $\theta_{i}$ are in terms of $(1-\Pi(M))$, the computation in proof of Theorem 10 also provides us with a bound on $(1-\Pi(M))$. Note that we can also define $\theta_{i}^{\prime}$ using a slightly lose bound:

$$
\theta_{i}^{\prime}=\int_{u=G^{i-1}}^{G^{i-2}} \lambda_{i-1} \theta_{i-1} e^{-\lambda_{i-1} u} d u=\theta_{i-1}\left[e^{-\lambda_{i-1} G^{i-1}}-e^{-\lambda_{i-1} G^{i-2}}\right]
$$

(this is exactly what we have done in Theorem 8). This gives a simple representation of $\theta_{i}$ as a recursive product of exponentials of some lengths $l_{i}$ as in Theorem 8 .

\section{Numerical Results}

We obtained time average density of the window process from $n s-2$ [33] simulations for AIMD protocol with constant loss rate and MIMD protocol with linear loss rate. The multiplicative decrease factor $\beta=0.5$ for both the protocols and the loss rate, $\lambda_{a}$, for AIMD protocol was set to either 0.005 or 0.008 . The MIMD protocol had an increase profile of $f_{m}(x)=1.01 x$ as in Scalable TCP while the AIMD protocol had $f_{a}(x)=1$. The loss rate for MIMD protocol was $\lambda(x)=\lambda_{m} x$ where $\lambda_{m}$ was chosen so that the conditions of Theorem 1 were satisfied. This requirement is satisfied if $\lambda_{m}=0.01 \lambda_{a}$, i.e., $\lambda_{m}=0.00005$ or 0.00008 . Figure 5 gives 
the function $\pi_{m}(x)$ for MIMD and $\frac{C f_{a}(x) \pi_{a}(x)}{f_{m}(x)}$ where $C$ is $\frac{\lambda_{m} E_{m}[X]}{\lambda_{a}}$ with $E_{m}[X]$ being the expected window size for MIMD protocol obtained from simulation. The results are as predicted by Theorem 1, i.e., $\pi_{m}(x)=\frac{\lambda_{m} E_{m}[X]}{\lambda_{a}}, \forall x$. For the same experimental setup, we

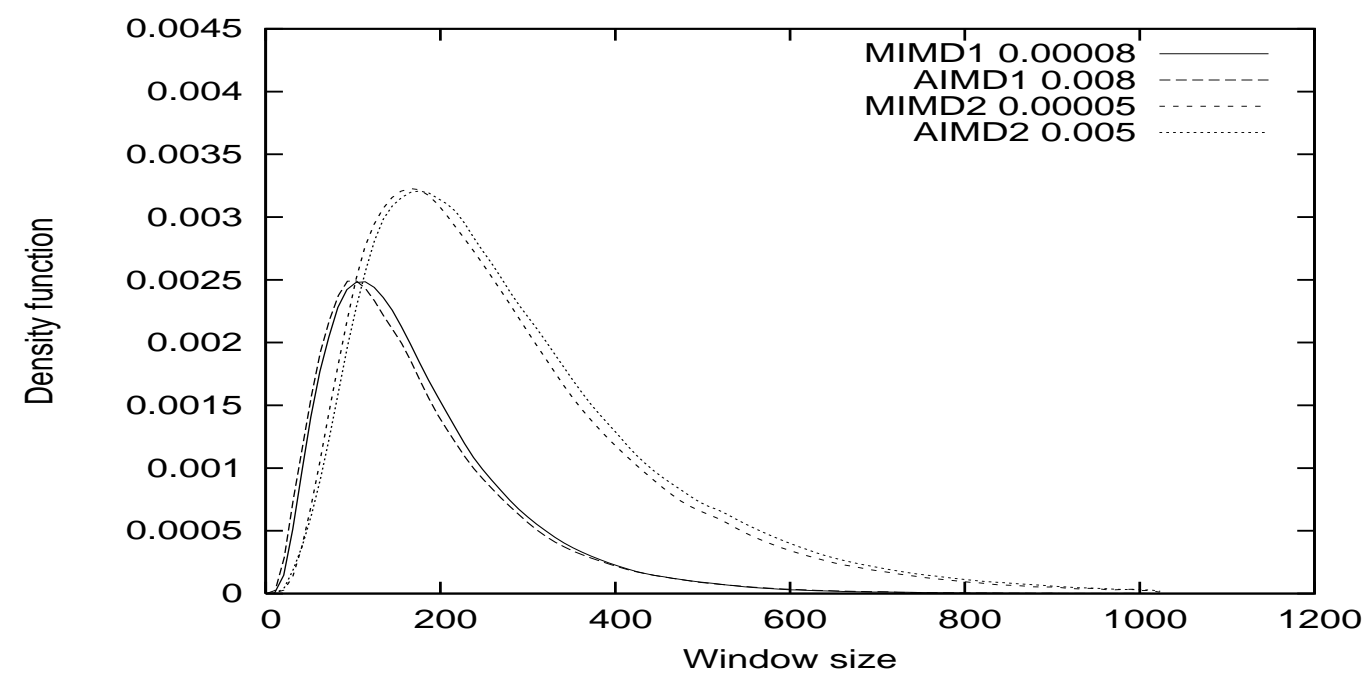

Figure 5: Comparison of time average distribution for MIMD with linear loss rate and for AIMD with constant loss rate.

also obtained the distribution of window sizes just before losses. The results are plotted in Figure 6 which shows that, in agreement with Theorem 2, this distribution is same for the two systems. Now, we compute the numerical values from our analysis of Section 6.2 and compare it with simulation results of Figure 5 for MIMD with linear loss rate. Figure 7 gives the comparison between analysis and simulations. Since the density function is already plotted in Figure 5, here we plot the $\left(E\left[X^{n}\right]\right)^{\frac{1}{n}}$ vs. $n$ for $1 \leq n \leq 10$. The analysis and simulations are seen to match well for smaller values of $n(\leq 6)$; the small discrepancy for large values of $n$ could be owing to finite simulation run-length.

Figure 8 gives results from simulation and numerical computation of analysis of Section 6.1.1 for TCP's standard AIMD protocol with linear loss rate for different values of $\lambda=1 e-5,2 e-5$ and $5 e-5$. The slight discrepancy between simulation and analytical results could be owing to numerical problems involved in solving the required recursions (see [5] for discussion on similar lines).

Figure 9 gives complementary distribution function of the stationary window process for HSTCP assuming that the multiplicative decrease factor $b(w)$ is fixed to a constant value $B$. Recall the parameters $A, B, \mu$ and $\nu$ of Section 6.3. We fix $A=0, B=0.125$ and $\nu$ so that $\frac{2 B \nu}{2-B}=0.01$ so that the case of $\mu=-1$ corresponds to the Scalable TCP [8]. The plot shows results for values of the parameter $\mu=-0.9,-1.0,-1.2$. In order to do this, 


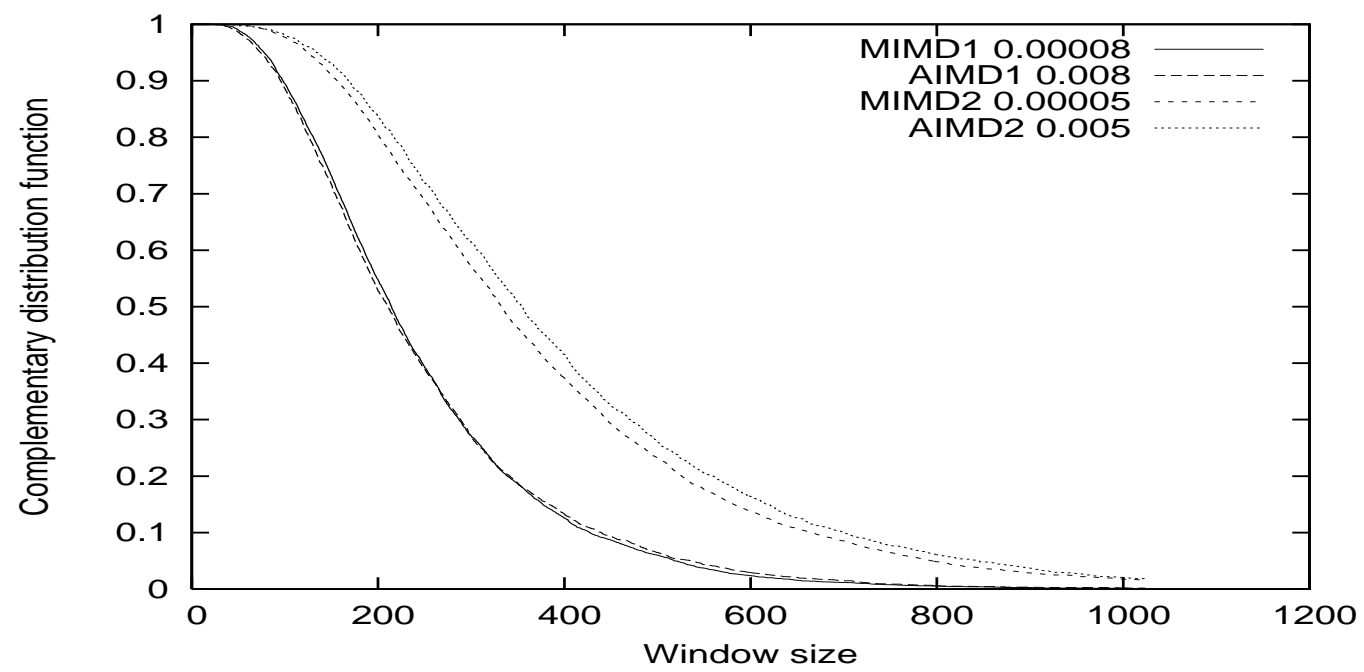

Figure 6: Window size distribution just before loss instants for MIMD with linear loss rate and for AIMD with constant loss rate.

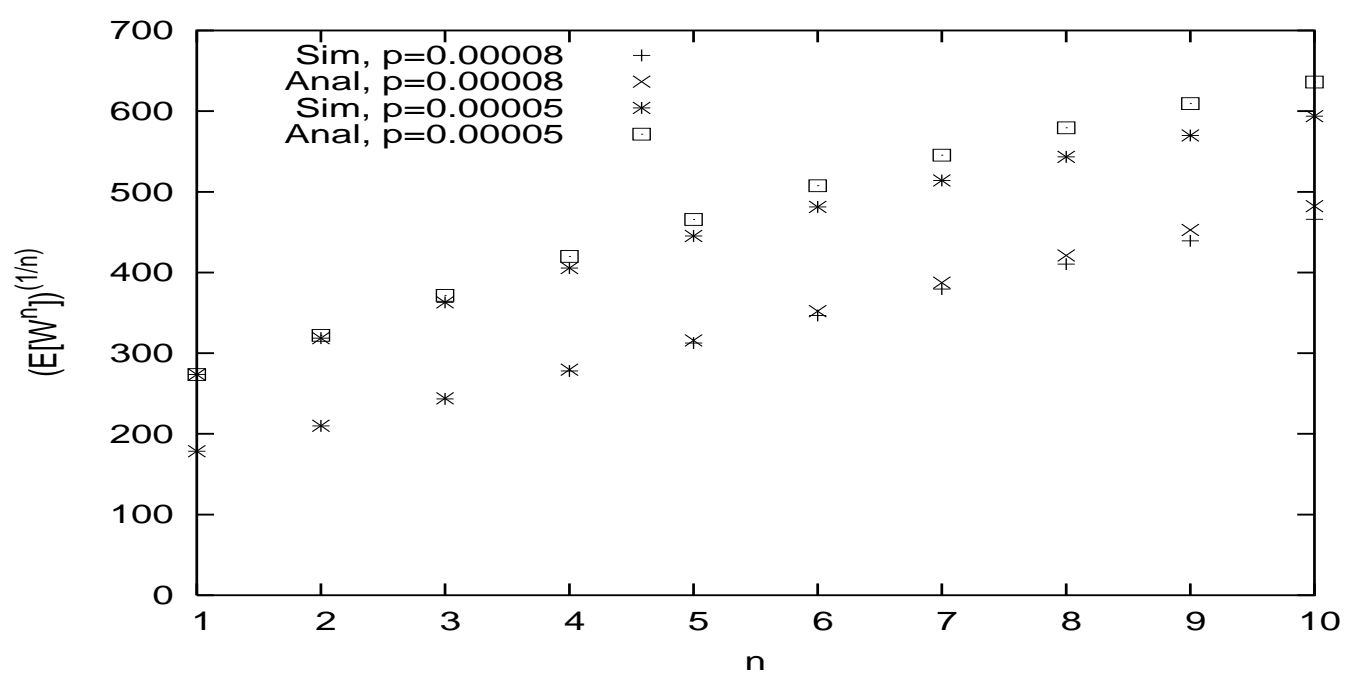

Figure 7: First 10 moments for MIMD with linear loss rate.

we varied the parameters $p_{l}$ and $p_{h}$ accordingly. The figure also gives numerical results from the analysis of Section 6.3. It is observed from the figure that one can approximate 


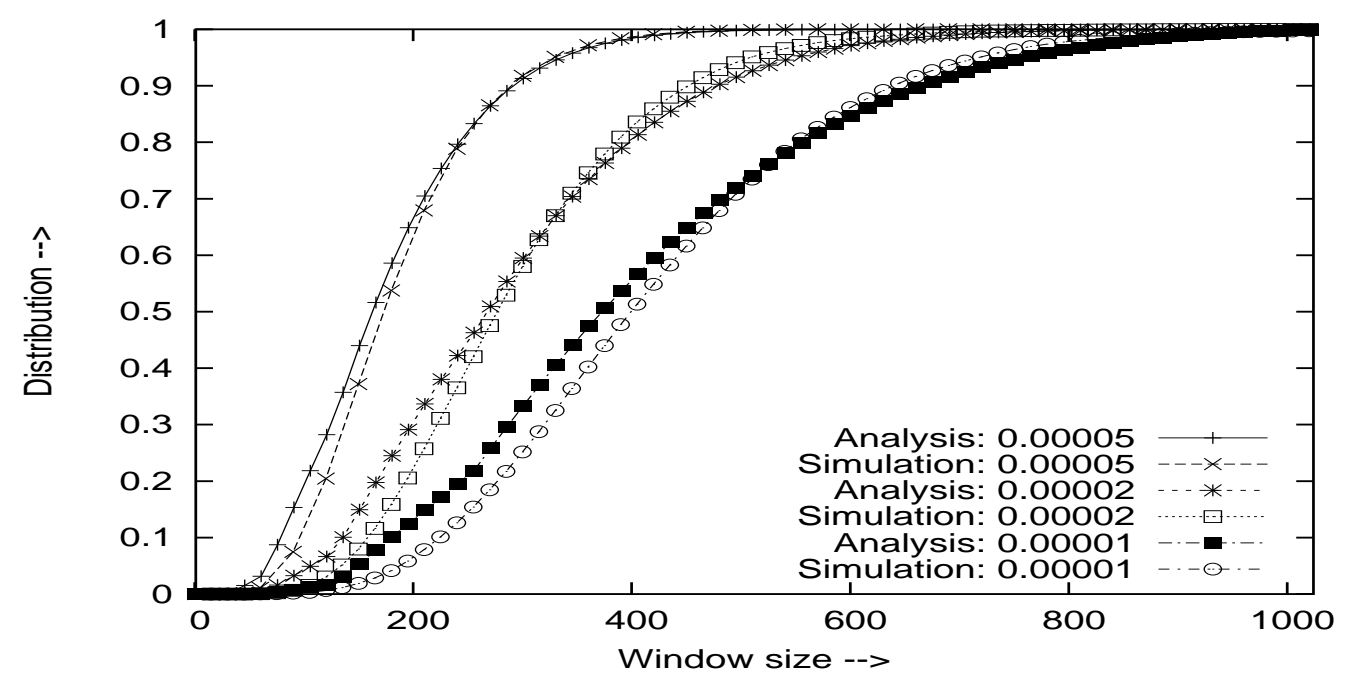

Figure 8: Window distribution for AIMD protocol with linear loss rate.

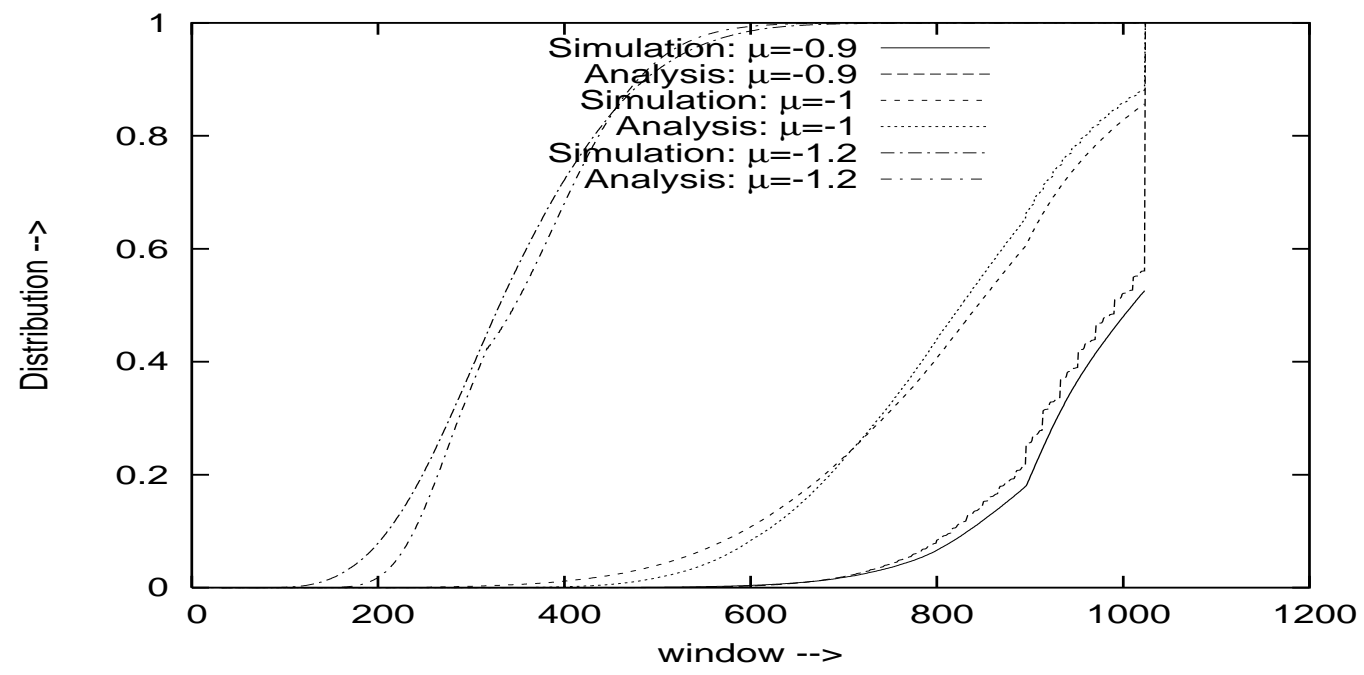

Figure 9: Window size distribution for HSTCP with linear loss rate.

any increase function only by varying $\mu$ while keeping the multiplicative drop factor $b(w)$ constant. This simplifies the algorithm as now there are not many independent design choices and, moreover, the analysis of Section 6.3 combined with that of [5] provides closed form 
result for the stationary distribution. We also note that the distribution is very sensitive to the value of the parameter $\mu$.

\section{Conclusion}

We considered a general congestion control protocol with a state dependent loss probability. We obtained closed for expression for the stationary window size distribution for a general AWP with a general loss rate. Various transformations introduced provided us with many equivalence relations. Most significant being that of the relation between window evolution and the workload process in a finite capacity queueing system with state dependent service and arrival rates and a state dependent deterministic service requirement. Several results of independent interest in queueing theory were obtained. Some monotonicity properties of the stationary window distribution as well as a necessary and sufficient condition for stability of the window size process were proved.

We have assumed that the loss $\lambda(\cdot)$ is a given function. This may be the case in the applications using AQM schemes and where congestion losses are rare. However, when most of the losses are owing to congestion losses, it appears to be more realistic that the form of $\lambda(\cdot)$ will itself be determined by the AWP. Also, it is possible that, like in model of [30], the loss process $\lambda(\cdot)$ may itself be a stochastic process. These considerations are topic of further research.

Theorem 7 may not be easily verifiable for a general AWP decrease profile (since this involves finding the functions $\left.J_{l}(\cdot)\right)$. A simpler condition to establish the convergence or divergence of the involved series is yet another further possible direction.

In the analysis of HSTCP we have chosen a multiplicative decrease algorithm with window independent decrease factor. We now aim at using some approximations for the evolution of the window process using the drop profile suggested in [14]. It is also important to study an optimal choice of the parameter $\mu$.

\section{A Justification of the Introduced Transformation}

In this appendix we give a justification of the transformation $x_{t} \rightarrow y_{t}$ introduced in Section 2.1. The evolution of the processes $\left\{x_{t}\right\}$ and $\left\{y_{t}\right\}$ are shown to be equivalent in the sense that the stationary distribution of one can be obtained from that of the other using the transformation of Section 2.1.

Let $\Pi_{t}(x) \triangleq P\left(x_{t} \leq x\right)$. Fix a $t$ and a small value $\Delta>0$. One can write the following evolution equation for the process $\left\{\Pi_{t}(\cdot)\right\}$,

$$
\Pi_{t+\Delta}(x)=\Pi_{t}(x-f(x) \Delta+o(\Delta))+\int_{u=x-f(x) \Delta+o(\Delta)}^{g^{-1}(x)} \lambda(u) \Delta d \Pi_{t}(u)+o(\Delta) .
$$

This evolution is also derived in Section 2.4. The first term on right hand side above is the fact that if $x_{t}<y$ such that $x=y+f(y) \Delta$ then $x_{t+\Delta} \leq x$ whether or not a loss event takes place in the interval $[t, t+\Delta]$. Using Taylor series expansion, it follows that

$\mathrm{RR} \mathrm{n}^{\circ} 5262$ 
$y=x-f(x) \Delta+o(\Delta)$. The second term on the right hand side above is the probability that a loss event takes place in $[t, t+\Delta]$ and results in a window size $x_{t+\Delta} \leq x$. The $o(\Delta)$ term collects the possibility of multiple loss events in the interval $[t, t+\Delta]$. Once again using the Taylor series expansion for the first term on right hand side, we get

$$
\begin{aligned}
\Pi_{t+\Delta}(x) & =\left[\Pi_{t}(x)-(f(x) \Delta+o(\Delta)) \frac{\partial \Pi_{t}(x)}{\partial x}+o(\Delta)\right]+\int_{u=x-f(x) \Delta+o(\Delta)}^{g^{-1}(x)} \lambda(u) \Delta d \Pi_{t}(u)+o(\Delta) \\
& =\Pi_{t}(x)-f(x) \Delta \frac{\partial \Pi_{t}(x)}{\partial x}+\int_{u=x-f(x) \Delta+o(\Delta)}^{g^{-1}(x)} \lambda(u) \Delta d \Pi_{t}(u)+o(\Delta),
\end{aligned}
$$

where now the residual terms from Taylor series expansion is also $o(\Delta)$ and is collected in the last term above. Rearranging the above and dividing throughout by $\Delta$, we get

$$
\frac{\Pi_{t+\Delta}(x)-\Pi_{t}(x)}{\Delta}+f(x) \frac{\partial \Pi_{t}(x)}{\partial x}=\int_{u=x-f(x) \Delta+o(\Delta)}^{g^{-1}(x)} \lambda(u) d \Pi_{t}(u)+\frac{o(\Delta)}{\Delta} .
$$

Now, letting $\Delta \downarrow 0$, we get the following

Theorem 11 The evolution of the marginal distributions $\left\{\Pi_{t}(\cdot)\right\}$ is described by the following integro-differential equation

$$
\frac{\partial \Pi_{t}(x)}{\partial t}+f(x) \frac{d \Pi_{t}(x)}{d x}=\int_{u=x}^{g^{-1}(x)} \lambda(u) d \Pi_{t}(u) .
$$

Assuming $\left\{\Pi_{t}(\cdot)\right\}$ admits a limit in the steady state, we can substitute the time derivative in Equation 14 by 0 . Letting $\Pi(\cdot)$ denote the achieved limit, i.e., the steady state distribution for the process $\left\{x_{t}\right\}$, we see that

$$
f(x) \frac{d \Pi(x)}{d x}=\int_{u=x}^{g^{-1}(x)} \lambda(u) d \Pi(u) .
$$

Remark: We remark here that the relation given by Equation 15 is frequently met with in various studies concerning evolution of dynamical systems. These equation are also called the Fredholm equation of second kind and the existence of its solution is now a well studied topic. (The Fredholm equations require the limits of integration to be fixed; this is easily incorporated here by use of the indicator functions and taking the range of integration to be $[0, \infty)$.)

Let us now consider the transformation given by $x \mapsto F(x) \triangleq y$ introduced in Section 2.1. Let $\tilde{\Pi}_{t}(y) \triangleq \Pi_{t}\left(F^{-1}(y)\right)$ or, $\Pi(x)=\tilde{\Pi}_{t}(F(x))$. Equation 13 now becomes

$$
\frac{\tilde{\Pi}_{t+\Delta}(y)-\tilde{\Pi}_{t}(y)}{\Delta}+\frac{\partial \tilde{\Pi}_{t}(y)}{\partial y}=\int_{u=F(x-f(x) \Delta+o(\Delta))}^{F\left(g^{-1}(x)\right)} \lambda(F(u)) d \tilde{\Pi}_{t}(u)+\frac{o(\Delta)}{\Delta} .
$$


Now, $F(x-f(x) \Delta+o(\Delta))=F(x)-f(x) F^{\prime}(x) \Delta+o(\Delta)=y-\Delta+o(\delta)$. Hence we get, with $\tilde{\lambda}(u) \triangleq \lambda(F(u))$,

$$
\frac{\tilde{\Pi}_{t+\Delta}(y)-\tilde{\Pi}_{t}(y)}{\Delta}+\frac{\partial \tilde{\Pi}_{t}(y)}{\partial y}=\int_{u=y-\Delta+o(\Delta))}^{F\left(g^{-1}\left(F^{-1}(y)\right)\right)} \tilde{\lambda}(u) d \tilde{\Pi}_{t}(u)+\frac{o(\Delta)}{\Delta} .
$$

Now, $F(x-f(x) \Delta+o(\Delta))=F(x)-f(x) F^{\prime}(x) \Delta+o(\Delta)=y-\Delta+o(\delta)$. Hence we get, with $\tilde{\lambda}(u) \triangleq \lambda(F(u))$,

$$
\frac{\tilde{\Pi}_{t+\Delta}(y)-\tilde{\Pi}_{t}(y)}{\Delta}+\frac{\partial \tilde{\Pi}_{t}(y)}{\partial y}=\int_{u=y-\Delta+o(\Delta))}^{F\left(g^{-1}\left(F^{-1}(y)\right)\right)} \tilde{\lambda}(u) d \tilde{\Pi}_{t}(u)+\frac{o(\Delta)}{\Delta} .
$$

Now letting $\Delta \downarrow 0$, we see that the time evolution of the process $\left\{\tilde{\Pi}_{t}(\cdot)\right\}$ is given by an expression similar to that for $\left\{\Pi_{t}(\cdot)\right\}$ as in Theorem 11, i.e.,

$$
\frac{\partial \tilde{\Pi}_{t}(y)}{\partial t}+\frac{\partial \tilde{\Pi}_{t}(y)}{\partial y}=\int_{u=y}^{G^{-1}(y)} \tilde{\lambda}(u) d \tilde{\Pi}_{t}(u),
$$

where $G^{-1}(y) \triangleq F\left(g^{-1}\left(F^{-1}(y)\right)\right)$, i.e., $y=G\left(F\left(g^{-1}\left(F^{-1}(y)\right)\right)\right)$.

We note that Equation 19 is the same as Equation 14 with $f(\cdot)$ replaced by a function identically equal to unity, $g(\cdot)$ replaced by $G(\cdot)$ and $\lambda(\cdot)$ substituted by $\tilde{\lambda}(\cdot)$. This new system corresponds to the case where the process $\left\{y_{t}\right\}$ is linearly increasing with time between the loss instants and drops to a value $G\left(y_{t}\right)$ when loss occurs at time $t$. We also note that, owing to the fact that $F(\cdot)$ is invertible, there is a one to one correspondence between the processes $\left\{x_{t}\right\}$ and $\left\{y_{t}\right\}$. Thus it is enough to study the process $\left\{y_{t}\right\}$ to study the properties of process $\left\{x_{t}\right\}$. This transformation is useful because now the process $\left\{y_{t}\right\}$ is determined only by two functions $\tilde{\lambda}(\cdot)$ and $G(\cdot)$.

\section{B Proof of Theorem 4}

The Kolmogorov equation for this system is

$$
\pi(x)=\left\{\begin{array}{lc}
\int_{u=x}^{H(x)} \pi(u) d u & x \in I_{k}, k \geq 2, \\
\int_{u=x}^{M-x} \pi(u) d u+P_{M} & x \in I_{1}
\end{array}\right.
$$

where $M=G^{0}, G^{i}=G\left(G^{i-1}\right)$ and $I_{k}=\left[G^{k}, G^{k+1}\right]$. Let $\Pi^{c}(x)$ denote the tail distribution function. For $x \in I_{k}, k \geq 2$,

$$
\begin{array}{r}
\frac{d}{d x} \Pi^{c}(x)=\Pi^{c}(H(x))-\Pi^{c}(x) \\
\frac{d}{d x} e^{x} \Pi^{c}(x)=e^{x} \Pi^{c}(H(x))
\end{array}
$$

$\mathrm{RR} \mathrm{n}^{\circ} 5262$ 


$$
\begin{aligned}
\frac{d}{d x} e^{x} \Pi^{c}(x) & =e^{x-H(x)} e^{H(x)} \Pi^{c}(H(x)) \\
\frac{d}{d x} e^{x} \Pi^{c}(x) & =e^{-D(x)} e^{H(x)} \Pi^{c}(H(x))
\end{aligned}
$$

where $D(x)=H(x)-x$ is the downward jump at $H(x)$. Similarly, for $x>G^{1}$,

$$
\begin{array}{r}
-\frac{d}{d x} \Pi^{c}(x)=\int_{u=x}^{M-} \pi(u) d u+P_{M} \\
-\frac{d}{d x} \Pi^{c}(x)=\Pi^{c}(x) \\
\frac{d}{d x} e^{x} \Pi^{c}(x)=0 \\
e^{x} \Pi^{c}(x)=c_{1}
\end{array}
$$

for $c_{1}=e^{G^{1}} \Pi^{c}\left(G^{1}\right)$. Note that we also have that $P_{M}=\Pi^{c}(M)=c_{1} e^{-M}$. Thus, for $x \in I_{2}$,

$$
\begin{aligned}
\frac{d}{d x} e^{x} \Pi^{c}(x) & =e^{-D(x)} e^{H(x)} \Pi^{c}(H(x)) \\
& =e^{-D(x)} c_{1} \\
\Pi^{c}(x) e^{x} & =c_{2}+c_{1} \int_{u=G^{2}}^{x} e^{-D(u)} d u \\
& =c_{2}+c_{1} J_{2,1}(x) .
\end{aligned}
$$

For $x \in I_{3}$,

$$
\begin{aligned}
\frac{d}{d x} e^{x} \Pi^{c}(x) & =e^{-D(x)} e^{H(x)} \Pi^{c}(H(x)) \\
& =e^{-D(x)}\left[c_{2}+c_{1} J_{2,1}(H(x))\right] \\
e^{x} \Pi^{c}(x) & =c_{3}+c_{2} \int_{u=G^{3}}^{x} e^{-D(u)} d u+c_{1} \int_{u=G^{3}}^{x} e^{-D(u)} J_{2,1}(H(u)) d u \\
& =c_{3}+c_{2} J_{3,1}(x)+c_{1} J_{3,2}(x) .
\end{aligned}
$$

Proceeding in this way, we see that for $x \in I_{k}, k \geq 2$,

$$
e^{x} \Pi^{c}(x)=\sum_{j=1}^{k} c_{j} J_{k, k-j}(x)
$$

where $J_{k, l}(x)=\int_{G^{k}}^{x} e^{-D(x)} J_{k-1, l-1}(H(u)) d u$ and $c_{k}=e^{G^{k}} \Pi^{c}\left(G^{k}\right)$.

Now, using continuity of $\Pi^{c}(x)$ at the boundary $G^{k-1}$, we get

$$
\sum_{j=1}^{k} c_{j} J_{k, k-j}\left(G^{k-1}\right)=\sum_{j=1}^{k-1} c_{j} J_{k-1, k-1-j}\left(G^{k-1}\right)=c_{k-1}
$$

INRIA 


$$
c_{k}=\sum_{j=1}^{k-1} c_{j}\left[J_{k-1, k-1-j}\left(G^{k-1}\right)-J_{k, k-j}\left(G^{k-1}\right)\right]=\sum_{j=1}^{k-1} c_{j} q_{k, j}
$$

where $J_{k-1, k-1-j}\left(G^{k-1}\right)-J_{k, k-j}\left(G^{k-1}\right)=q_{k, j}$. Now, since $J_{k-1, k-1-j}\left(G^{k-1}\right)=0$ for $j<k-1$ and $J_{k-1,0}\left(G^{k-1}\right)=1$, it is seen that $q_{k, j}=-J_{k, k-j}\left(G^{k-1}\right)$ for $j<k-1$ and $q_{k, k-1}=1-J_{k, 1}\left(G^{k-1}\right)$. Thus $c_{k}$ can be written in terms of $c_{1}$ as

$$
c_{k}=c_{1} \sum_{j_{1}=1}^{k-1} \sum_{j_{2}=1}^{j_{1}-1} \ldots \sum_{j_{k-2}=1}^{j_{k-3}-1} q_{k, j_{1}} q_{j_{1}, j_{2}} \ldots q_{j_{k-2}, 1} .
$$

Now, since the lower bound is $G^{m}$ for some $m>1$, we get that $\Pi^{c}\left(G^{m}\right)=1$, hence,

$$
P_{M} e^{M}=c_{1}=\left[\sum_{j_{1}=1}^{m-1} \sum_{j_{2}=1}^{j_{1}-1} \ldots \sum_{j_{m-2}=1}^{j_{m-3}-1} q_{m, j_{1}} q_{j_{1}, j_{2}} \ldots q_{j_{m-2}, 1}\right]^{-1} .
$$

We have proved Theorem 4.

\section{Proof of Proposition 1}

It is seen that the Kolmogorov equation can be written as, for $k \leq m-2$ :

$$
\begin{aligned}
\Pi_{k}^{\prime}(y)= & \lambda\left(\Pi_{k+1}(y)-\Pi_{k}(y)\right) \\
\frac{d}{d y} \Pi_{k}(y) e^{\lambda y}= & \lambda e^{\lambda y} \Pi_{k+1}(y) \\
e^{\lambda y} \Pi_{k}(y)= & \lambda \int_{u_{1}=0+}^{y} e^{\lambda u_{1}} \Pi_{k+1}\left(u_{1}\right) d u_{1}+\Pi_{k}(0) \\
= & \lambda \int_{u_{1}=0+}^{y} e^{\lambda u_{1}} \Pi_{k+1}\left(u_{1}\right) d u_{1}+\Pi_{k}(0) \\
= & \lambda \int_{u_{1}=0+}^{y}\left[\lambda \int_{u_{2}=0+}^{u_{1}} e^{\lambda u_{2}} \Pi_{k+2}\left(u_{2}\right) d u_{2}+\Pi_{k+1}(0)\right] d u_{1}+\Pi_{k}(0) \\
= & \lambda \int_{u_{1}=0+}^{y}\left[\lambda \int_{u_{2}=0+}^{u_{1}}\left[\lambda \int_{u_{3}=0+}^{u_{2}} e^{\lambda u_{3}} \Pi_{k+3}\left(u_{3}\right) d u_{3}+\Pi_{k+2}(0)\right] d u_{2}+\Pi_{k+1}(0)\right] d u_{1} \\
& +\Pi_{k}(0) \\
=\lambda^{i} \int_{u_{1}=0+}^{y} \int_{u_{2}=0+}^{u_{1}} & \ldots \int_{u_{i}=0+}^{u_{i-1}} e^{\lambda u_{i}} \Pi_{k+i}\left(u_{i}\right) d u_{i} \ldots d u_{2} d u_{1}+\sum_{j=0}^{i-1} \Pi_{k+j}(0) \frac{(\lambda y)^{j}}{j !} \text { for } k+i \leq m-1 .
\end{aligned}
$$

In particular, for $i=m-k-1$, we get

$e^{\lambda y} \Pi_{k}(y)=\lambda^{m-k-1} \int_{u_{1}=0+}^{y} \int_{u_{2}=0+}^{u_{1}} \ldots \int_{u_{m-k-1}=0+}^{u_{m-k-2}} e^{\lambda u_{m-k-1}} \Pi_{m-1}\left(u_{m-k-1}\right) d u_{m-k-1} \ldots d u_{2} d u_{1}$ 


$$
+\sum_{j=0}^{m-k-2} \Pi_{k+j}(0) \frac{(\lambda y)^{j}}{j !} .
$$

For $(m-1) \theta \leq y$, the up and down crossing rates are equated as follows:

$$
\begin{aligned}
\Pi^{\prime}(y) & =\lambda \int_{u=y+}^{\infty} \pi(u) d u \\
\Pi^{\prime}(y) & =\lambda(1-\Pi(y)) \\
\Pi_{m-1}^{\prime}(y) & =\lambda\left(1-\Pi_{m-1}(y)\right) \\
\frac{d}{d y} \Pi_{m-1}(y) e^{\lambda y} & =\lambda e^{\lambda y} \\
\Pi_{m-1}(y) e^{\lambda y} & =\int_{u=0}^{y} \lambda e^{\lambda u} d u+\Pi_{m-1}(0) \\
& =e^{\lambda y}-1+\Pi_{m-1}(0) .
\end{aligned}
$$

Substituting this in Equation 20, we get,

$$
\begin{aligned}
e^{\lambda y} \Pi_{k}(y)= & \lambda^{m-k-1} \int_{u_{1}=0+}^{y} \int_{u_{2}=0+}^{u_{1}} \ldots \int_{u_{m-k-1}=0+}^{u_{m-k-2}} e^{\lambda u_{m-k-1}} d u_{m-k-1} \ldots d u_{2} d u_{1} \\
& +\sum_{j=0}^{m-k-1} \Pi_{k+j}(0) \frac{(\lambda y)^{j}}{j !}-\frac{(\lambda y)^{m-k-1}}{(m-k-1) !} \\
= & e^{\lambda y}-\sum_{j=0}^{m-k-1}\left(1-\Pi_{k+j}(0)\right) \frac{(\lambda y)^{j}}{j !}
\end{aligned}
$$

Now, noting that $\Pi_{k}(0)=\Pi_{k-1}(\theta)$ and that $\Pi(0)=\Pi_{0}(0)=0$, and integrating the above, we get a value of $\Pi((m-1) \theta)$.

The original system of MIMD can be obtained by the reverse transformation. Proposition 1 thus follows.

\section{Proof of Proposition 2}

We know from Equation 21 that

$$
e^{\lambda y} \Pi_{k}^{c}(y)=\sum_{j=0}^{m-k-1} \Pi_{k+j}^{c}(0) \frac{(\lambda y)^{j}}{j !}, \quad k \leq m-2 .
$$

Let $F_{k} \triangleq \Pi_{k}^{c}(0)$. From continuity of $\Pi(\cdot)$, it follows that

$$
F_{k+1}=1-\Pi_{k+1}(0)=1-\Pi_{k}(\theta) .
$$

INRIA 
Thus,

$$
\begin{aligned}
e^{\lambda \theta} F_{k+1} & =\sum_{j=0}^{m-k-1} F_{k+j} \frac{(\lambda \theta)^{j}}{j !} \\
\Rightarrow F_{k} & =a F_{k+1}-\sum_{j=1}^{m-k-1} F_{k+j} \frac{b^{j}}{j !}, \quad k \leq m-2,
\end{aligned}
$$

where $b=\lambda \theta$ and $a=e^{b}$. The above relation can be applied again to get

$$
\begin{aligned}
F_{k} & =a F_{k+1}-\sum_{j=1}^{m-k-1} F_{k+j} \frac{b^{j}}{j !} \\
& =a^{2} F_{k+2}-a \sum_{j=1}^{m-k-2} F_{k+1+j} \frac{b^{j}}{j !}-\sum_{j=1}^{m-k-1} F_{k+j} \frac{b^{j}}{j !} \\
& =a^{l} F_{k+l}-\sum_{s=0}^{l-1} a^{s} \sum_{j=1}^{m-k-1-s} F_{k+s+j} \frac{b^{j}}{j !}, \quad l \leq m-k-1,(k+l-1 \leq m-2) \\
& =a^{m-k-1} F_{m-1}-\sum_{s=0}^{m-k-2} a^{s} \sum_{j=1}^{m-k-1-s} F_{k+s+j} \frac{b^{j}}{j !} \\
& =a^{m-k-1} F_{m-1}-\sum_{l=1}^{m-k-1} F_{k+l} \sum_{j=1}^{l} a^{l} \frac{\left(\frac{b}{a}\right)^{j}}{j !} \\
& =a^{m-k-1} F_{m-1}-\sum_{l=1}^{m-k-1} F_{k+l} \phi_{1}(l) \\
& =\left(a^{m-k-1}-\phi_{1}(m-k-1)\right) F_{m-1}-\sum_{l=1}^{m-k-2} F_{k+l} \phi_{1}(l)
\end{aligned}
$$

where $\phi_{1}(l) \triangleq \sum_{j=1}^{l} a^{l} \frac{\frac{b}{a}^{j}}{j !}$ is independent of $k$. Note that $\phi_{1}(1)=b$, implying $F_{m-2}=$ $F_{m-1}(a-b)$. Using Equation 22 again, we get, for $k \leq m-3$,

$$
\begin{aligned}
F_{k}= & \left(a^{m-k-1}-\phi_{1}(m-k-1)\right) F_{m-1}-\sum_{l=1}^{m-k-2} \phi_{1}(l)\left[\left(a^{m-k-l-1}-\phi_{1}(m-k-l-1)\right) F_{m-1}-\right. \\
& \left.\sum_{s=1}^{m-k-l-2} F_{k+l+s} \phi_{1}(s)\right] \\
= & F_{m-1}\left[\left(a^{m-k-1}-\phi_{1}(m-k-1)\right)-\sum_{l=1}^{m-k-2} \phi_{1}(l)\left(a^{m-k-l-1}-\phi_{1}(m-k-l-1)\right)\right]+
\end{aligned}
$$

$\mathrm{RR} \mathrm{n}^{\circ} 5262$ 


$$
\begin{aligned}
& \sum_{l=1}^{m-k-2} \phi_{1}(l) \sum_{s=1}^{m-k-l-2} F_{k+l+s} \phi_{1}(s) \\
& =F_{m-1}\left[\left(a^{m-k-1}-\phi_{1}(m-k-1)\right)-\sum_{l=1}^{m-k-2} \phi_{1}(l)\left(a^{m-k-l-1}-\phi_{1}(m-k-l-1)\right)\right]+ \\
& \sum_{l=1}^{m-k-3} \phi_{1}(l) \sum_{s=1}^{m-k-l-2} F_{k+l+s} \phi_{1}(s) \\
& =F_{m-1}\left[\left(a^{m-k-1}-\phi_{1}(m-k-1)\right)-\sum_{l=1}^{m-k-2} \phi_{1}(l)\left(a^{m-k-l-1}-\phi_{1}(m-k-l-1)\right)\right]+ \\
& \sum_{l=2}^{m-k-2} F_{k+l} \sum_{s=1}^{l-1} \phi_{1}(l-s) \phi_{1}(s) \\
& =F_{m-1}\left[\left(a^{m-k-1}-\phi_{1}(m-k-1)\right)-\sum_{l=1}^{m-k-2} \phi_{1}(l)\left(a^{m-k-l-1}-\phi_{1}(m-k-l-1)\right)\right]+ \\
& \sum_{l=2}^{m-k-2} F_{k+l} \phi_{2}(l) \\
& =F_{m-1}\left[\left(a^{m-k-1}-\phi_{1}(m-k-1)\right)-\sum_{l=1}^{m-k-2} \phi_{1}(l)\left(a^{m-k-l-1}-\phi_{1}(m-k-l-1)\right)\right]+ \\
& \sum_{l=2}^{m-k-2} \phi_{2}(l)\left[\left(a^{m-k-l-1}-\phi_{1}(m-k-l-1)\right) F_{m-1}-\sum_{s=1}^{m-k-l-2} F_{k+l+s} \phi_{1}(s)\right] \\
& =F_{m-1}\left[\left(a^{m-k-1}-\phi_{1}(m-k-1)\right)-\sum_{l=1}^{m-k-2} \phi_{1}(l)\left(a^{m-k-l-1}-\phi_{1}(m-k-l-1)\right)+\right. \\
& \left.\sum_{l=2}^{m-k-2} \phi_{2}(l)\left(a^{m-k-l-1}-\phi_{1}(m-k-l-1)\right)\right]-\sum_{l=2}^{m-k-2} \phi_{2}(l) \sum_{s=1}^{m-k-l-2} F_{k+l+s} \phi_{1}(s) \\
& =F_{m-1}\left[\left(a^{m-k-1}-\phi_{1}(m-k-1)\right)-\sum_{l=1}^{m-k-2} \phi_{1}(l)\left(a^{m-k-l-1}-\phi_{1}(m-k-l-1)\right)+\right. \\
& \left.\sum_{l=2}^{m-k-2} \phi_{2}(l)\left(a^{m-k-l-1}-\phi_{1}(m-k-l-1)\right)\right]-\sum_{l=2}^{m-k-3} \phi_{2}(l) \sum_{s=1}^{m-k-l-2} F_{k+l+s} \phi_{1}(s) \\
& =F_{m-1}\left[\left(a^{m-k-1}-\phi_{1}(m-k-1)\right)-\sum_{l=1}^{m-k-2} \phi_{1}(l)\left(a^{m-k-l-1}-\phi_{1}(m-k-l-1)\right)+\right.
\end{aligned}
$$

INRIA 


$$
\begin{aligned}
& \left.\sum_{l=2}^{m-k-2} \phi_{2}(l)\left(a^{m-k-l-1}-\phi_{1}(m-k-l-1)\right)\right]-\sum_{l=3}^{m-k-2} F_{k+l} \sum_{s=1}^{l-2} \phi_{2}(l-s) \phi_{1}(s) \\
= & F_{m-1}\left[\left(a^{m-k-1}-\phi_{1}(m-k-1)\right)-\sum_{l=1}^{m-k-2} \phi_{1}(l)\left(a^{m-k-l-1}-\phi_{1}(m-k-l-1)\right)+\right. \\
& \left.\sum_{l=2}^{m-k-2} \phi_{2}(l)\left(a^{m-k-l-1}-\phi_{1}(m-k-l-1)\right)\right]-\sum_{l=3}^{m-k-2} F_{k+l} \phi_{3}(l) \\
= & F_{m-1}\left[\left(a^{m-k-1}-\phi_{1}(m-k-1)\right)+\sum_{s=1}^{j}(-1)^{s} \sum_{l=s}^{m-k-2} \phi_{s}(l)\left(a^{m-k-l-1}-\phi_{1}(m-k-l-1)\right)\right]+ \\
= & F_{m-1}\left[\left(a^{m-k-1}-\phi_{1}(m-k-1)\right)+\sum_{s=1}^{j+1} \sum_{l=j+1}^{m-k-2} F_{k+l} \phi_{j+1}(l), \quad j+1 \leq \sum_{l=s}^{m-k-2} \phi_{s}(l)\left(a^{m-k-l-1}-\phi_{1}(m-k-l-1)\right)\right]+ \\
& (-1)^{m-k-2} F_{m-2} \phi_{m-k-2}(m-k-2) \\
\Rightarrow & F_{m-1}\left[\left(a^{m-k-1}-\phi_{1}(m-k-1)\right)+\sum_{s=1}^{m-k-3}(-1)^{s} \sum_{l=s}^{m-k-2} \phi_{s}(l)\left(a^{m-k-l-1}-\phi_{1}(m-k-l-1)\right)+\right. \\
& \left.(-1)^{m-k-2}(a-b) \phi_{m-k-2}(m-k-2)\right] .
\end{aligned}
$$

Here,

$$
\phi_{j+1}(l)=\sum_{s=1}^{l-j} \phi_{j}(l-s) \phi_{1}(s), \quad j \geq 1 .
$$

The above expression for $F_{k}$ is valid for $k \leq m-2$ if we assume that $\phi_{0}(0)=0$. Now, since $F_{0}=1$, we see that

$$
\begin{aligned}
1= & F_{m-1}\left[\left(a^{m-1}-\phi_{1}(m-1)\right)+\sum_{s=1}^{m-3}(-1)^{s} \sum_{l=s}^{m-2} \phi_{s}(l)\left(a^{m-l-1}-\phi_{1}(m-l-1)\right)+\right. \\
& \left.(-1)^{m-2}(a-b) \phi_{m-2}(m-2)\right] \\
F_{m-1}= & {\left[\left(a^{m-1}-\phi_{1}(m-1)\right)+\sum_{s=1}^{m-3}(-1)^{s} \sum_{l=s}^{m-2} \phi_{s}(l)\left(a^{m-l-1}-\phi_{1}(m-l-1)\right)+\right.} \\
& \left.(-1)^{m-2}(a-b) \phi_{m-2}(m-2)\right]^{-1} .
\end{aligned}
$$

This proves Proposition 2.

$\mathrm{RR} \mathrm{n}^{\circ} 5262$ 


\section{E Proof of Proposition 3}

For this case, the Kolmogorov equations are

$$
\begin{aligned}
\pi(x) & =\int_{u=x}^{H(x)} \pi(u) \lambda u d u, \quad x_{\min } \leq x<G^{1}, \\
& =\int_{u=x}^{G^{0}} \pi(u) \lambda u d u+\int_{u=G^{0}}^{\infty} \pi(u) \lambda G^{0} d u \quad G^{1}<x \leq G^{0}, \\
& =\int_{u=x}^{\infty} \pi(u) \lambda G^{0} d u \quad x \geq G^{0} .
\end{aligned}
$$

We will obtain the expressions for $\pi(\cdot)$ and $\Pi(\cdot)$ over the interval $\left[G^{i}, G^{i-1}\right]$ using recursion on $i$.

We first consider the case $x>G^{0}$. For this case the Kolmogorov equation is

$$
\Pi^{\prime}(x)=\int_{u=x}^{\infty} \lambda G^{0} \pi(u) d u=\lambda G^{0}[1-\Pi(x)] .
$$

Multiplying both side of above by $e^{\lambda G^{0} x}$, we observe that, after some rearrangement,

$$
\frac{d}{d x} \Pi(x) e^{\lambda G^{0} x}=\lambda G^{0} e^{\lambda G^{0} x} .
$$

Integrating both the sides above over the interval $\left[G^{0}, x\right]$ (since the above relation is true only for $x \geq G^{0}$, the lower limit of integration has to be at least $G^{0}$ ),

$$
e^{\lambda G^{0} x} \Pi(x)=\int_{u=G^{0}}^{x} \lambda G^{0} e^{\lambda G^{0} u} d u+e^{\lambda\left(G^{0}\right)^{2}} \Pi\left(G^{0}\right)
$$

where the last term is the integration constant. From this we see that

$$
\Pi(x)=1-e^{\lambda\left(G^{0}\right)^{2}-\lambda G^{0} x}\left(1-\Pi\left(G^{0}\right)\right),
$$

thus, for $\theta_{1}=\lambda G^{0} e^{\lambda\left(G^{0}\right)^{2}}\left(1-\Pi\left(G^{0}\right)\right)$,

$$
\pi(x)=\theta_{1} e^{-\lambda G^{0} x} .
$$

The Kolmogorov equation for $x \in\left[G^{1}, G^{0}\right]$ is

$$
\begin{aligned}
\pi(x) & =\int_{u=x}^{G^{0}} \pi(u) \lambda u d u+\int_{u=G^{0}}^{\infty} \pi(u) \lambda G^{0} d u=\int_{u=x}^{G^{0}} \lambda u \pi(u) d u+\lambda G^{0}\left[1-\Pi\left(G^{0}\right)\right] \\
& \triangleq \eta_{2}+\int_{u=x}^{G^{0}} \lambda u \pi(u) d u
\end{aligned}
$$

INRIA 
Since the integrand on the right hand side above has its argument in the interval $\left[x, G^{0}\right]$ (and hence is in interval $\left[G^{1}, G^{0}\right]$ ), we can use the above equation (with $x$ replaced by $u$ ) to substitute for $\pi(u)$ on the right hand side above. This can be repeated any number of times and we get that for $x \in\left[G^{1}, G^{0}\right]$,

$$
\pi(x)=\eta_{2}+\eta_{2} \sum_{j=1}^{\infty} \lambda^{j} \int_{u_{1}=x}^{G^{0}} \ldots \int_{u_{j}=u_{j-1}}^{G^{0}} u_{j} \ldots u_{1} d u_{j} \ldots d u_{1} .
$$

The general $j$-fold integral in the right hand side above can be evaluated as follows:

$\int_{u_{1}=x}^{G^{0}} \ldots \int_{u_{j}=u_{j-1}}^{G^{0}} u_{j} \ldots u_{1} d u_{j} \ldots d u_{1}=\left(\frac{G^{02}-x^{2}}{2}\right)^{j} \int_{u_{1}=x}^{G^{0}} \ldots \int_{u_{j}=u_{j-1}}^{G^{0}} \frac{u_{j}}{\frac{G^{0}-x^{2}}{2}} \ldots \frac{u_{1}}{\frac{G^{0}-x^{2}}{2}} d u_{j} \ldots d u_{1}$

The last integral above is then the probability of one of the possible $j$ ! orderings of the i.i.d. random variables $u_{i}$ each having a probability density of $\frac{u_{j}}{\frac{\left(G^{2}-x^{2}\right)}{2}}$ in the interval $\left[x, G^{0}\right]$.

Since all the possible $j$ ! orderings are equiprobable, the above integral is $\frac{1}{j !}$. We thus see that

$$
\int_{u_{1}=x}^{G^{0}} \ldots \int_{u_{j}=u_{j-1}}^{G^{0}} u_{j} \ldots u_{1} d u_{j} \ldots d u_{1}=\left(\frac{G^{0^{2}}-x^{2}}{2}\right)^{j} \frac{1}{j !} .
$$

The proof of first part thus follows by substituting the above expression in Equation 23.

We now consider the case where $x \in\left(G^{l}, G^{l-1}\right), m \geq l>1$. For this case the Kolmogorov equations were obtained before as

$$
\pi(x)=\int_{u=x}^{H(x)} \lambda u \pi(u) d u .
$$

Differentiating the above using the Liebnitz rule, we get that

$$
\pi^{\prime}(x)=\lambda H(x) \pi(H(x)) H^{\prime}(x)-\lambda x \pi(x) .
$$

Multiplying both side of above by $e^{\lambda \frac{x^{2}}{2}}$, we observe that, after some rearrangement,

$$
\frac{d}{d x} \pi(x) e^{\lambda \frac{x^{2}}{2}}=\lambda H(x) e^{\lambda \frac{x^{2}}{2}} \pi(H(x)) H^{\prime}(x) .
$$

Integrating both sides of the above, we see that

$$
\pi(x) e^{\lambda \frac{x^{2}}{2}}=\int_{u=x} \lambda H(u) e^{\lambda \frac{u^{2}}{2}} \pi(H(u)) H^{\prime}(u) d u+c_{l},
$$

for some appropriate integration constant $c_{l}$. A change of variable in the right hand side gives

$$
\begin{aligned}
\pi(x) e^{\lambda \frac{x^{2}}{2}} & =\int_{u_{1}=H(x)} \lambda u_{1} e^{\lambda \frac{G\left(u_{1}\right)^{2}}{2}} \pi\left(u_{1}\right) d u_{1}+c_{l} \\
& =\int_{u_{1}=H(x)} \lambda u_{1} e^{\lambda \frac{G\left(u_{1}\right)^{2}-u_{1}{ }^{2}}{2}} e^{\lambda \frac{u_{1}{ }^{2}}{2}} \pi\left(u_{1}\right) d u_{1}+c_{l}
\end{aligned}
$$

$\mathrm{RR} \mathrm{n}^{\circ} 5262$ 
Since the integrand on the right hand side above has its argument in the interval $\left[G^{l-1}, G^{l-2}\right]$, we can use the above equation (with $x$ replaced by $u$ and $l$ by $l-1$ ) to substitute for $\pi(u)$ on the right hand side above. It follows that

$$
\pi(x) e^{\lambda \frac{x^{2}}{2}}=\int_{u_{1}=H(x)} \lambda u_{1} e^{\lambda \frac{G\left(u_{1}\right)^{2}-u_{1}^{2}}{2}}\left[\int_{u_{2}=H\left(u_{1}\right)} \lambda u_{2} e^{\lambda \frac{G\left(u_{2}\right)^{2}-u_{2}{ }^{2}}{2}} e^{\lambda \frac{u_{2}^{2}}{2}} \pi\left(u_{2}\right) d u_{2}+c_{l-1}\right] d u_{1}+c_{l}
$$

Proceeding in a similar manner, the above can be seen to hold for any $0 \leq i \leq l-1$,

$$
\begin{aligned}
& \pi(x) e^{\lambda \frac{x^{2}}{2}}=\sum_{j=0}^{i-1} c_{l-j} \lambda^{j} \int_{u_{1}=H(x)} \ldots \int_{u_{j}=H\left(u_{j-1}\right)} u_{1} e^{\lambda \frac{G\left(u_{1}\right)^{2}-u_{1}{ }^{2}}{2}} u_{j} e^{\lambda \frac{G\left(u_{j}\right)^{2}-u_{j}{ }^{2}}{2}} d u_{j} \ldots d u_{1} \\
+ & \lambda^{i} \int_{u_{1}=H(x)} \ldots \int_{u_{i-1}=H\left(u_{i-2}\right)} \int_{u_{i}=H\left(u_{i-1}\right)} u_{1} e^{\lambda \frac{G\left(u_{1}\right)^{2}-u_{1}{ }^{2}}{2}} \ldots u_{i-1} e^{\lambda \frac{G\left(u_{i}\right)^{2}-u_{i}{ }^{2}}{2}} e^{\lambda \frac{u_{i}{ }^{2}}{2}} \pi\left(u_{i}\right) d u_{i} d u_{i-1} \ldots d u_{1}
\end{aligned}
$$

Above is true, in particular, for $G^{l-i}=G^{1}$, i.e., $i=l-1$. Thus we can use the fact that we know $\pi(x)$ for $x \in\left(G^{1}, G^{0}\right)$. Using $i=l-1$ and Equation 7 we get

$$
\pi(x) e^{\lambda \frac{x^{2}}{2}}=\sum_{j=0}^{l-1} c_{l-j} \lambda^{j} \int_{u_{1}=H(x)} \ldots \int_{u_{j}=H\left(u_{j-1}\right)} u_{1} e^{\lambda \frac{G\left(u_{1}\right)^{2}-u_{1}^{2}}{2}} \ldots u_{j} e^{\lambda \frac{G\left(u_{j}\right)^{2}-u_{j}{ }^{2}}{2}} d u_{j} \ldots d u_{1}
$$

Remark: Note that we are implicitly assuming that $u_{j} \in\left[G^{l-j}, G^{l-j-1}\right]$. This also requires that the constant $c_{j}$ should be appropriately chosen.

\section{F Proof of Proposition 4}

For the case of standard AIMD protocol, $G^{l}=\beta G^{l-1}$ for some $\beta<1$ such that $\beta$ is the (window independent) multiplicative decrease factor. Also $G(u)=\beta u$. This also means that $H(x)=\frac{x}{\beta}$.

$$
\pi(x) e^{\lambda \frac{x^{2}}{2}}=\sum_{j=0}^{l-1} c_{l-j} \lambda^{j} \int_{u_{1}=\frac{x}{\beta}} \ldots \int_{u_{j}=\frac{u_{j-1}}{\beta}} u_{1} e^{K u_{1}{ }^{2}} \ldots u_{j} e^{K u_{j}{ }^{2}} d u_{j} \ldots d u_{1}
$$

where $K=\lambda \frac{\beta^{2}-1}{2}$. We thus see that

$$
\begin{aligned}
& \pi(x) e^{\lambda \frac{x^{2}}{2}}=\sum_{j=0}^{l-1} c_{l-j} \lambda^{j} \frac{1}{2 K} \int_{u_{1}=\frac{x}{\beta}} \ldots \int_{u_{j-1}=\frac{u_{j-2}}{\beta}} u_{1} e^{K u_{1}{ }^{2}} \ldots u_{j-1} e^{K\left(1+\beta^{-2}\right) u_{j-1}{ }^{2}} d u_{j-1} \ldots d u_{1} \\
& =\sum_{j=0}^{l-1} c_{l-j} \lambda^{j} \frac{1}{2^{2} K^{2}\left(1+\beta^{-2}\right)} \int_{u_{1}=\frac{x}{\beta}} \ldots \int_{u_{j-2}=\frac{u_{j-3}}{\beta}} u_{1} e^{K u_{1}{ }^{2}} \ldots u_{j-2} e^{K\left(1+\beta^{-2}+\beta^{-4}\right) u_{j-2}{ }^{2}} d u_{j-2} \ldots d u_{1}
\end{aligned}
$$

INRIA 


$$
\begin{aligned}
& =\sum_{j=0}^{l-1} c_{l-j} \lambda^{j}\left(\Pi_{n=1}^{j} \frac{1}{2 K \sum_{\kappa=0}^{n} \beta^{-2 \kappa}}\right) e^{\frac{K}{\beta^{2}}\left(\sum_{\kappa=0}^{j-1} \beta^{-2 \kappa}\right) x^{2}} \\
\pi(x) & =\sum_{j=0}^{l-1} c_{l-j} b_{j} e^{a_{j} x^{2}}
\end{aligned}
$$

where $b_{j}=\Pi_{n=1}^{j} \frac{\lambda}{2 K \sum_{\kappa=0}^{n} \beta^{-2 \kappa}}$ and $a_{j}=\left(\frac{K}{\beta^{2}}\left(\sum_{\kappa=0}^{j-1} \beta^{-2 \kappa}\right)-0.5 \lambda\right)$, with $b_{0}=1$.

$$
\begin{aligned}
\pi(x) & =\int_{u=x}^{\frac{x}{\beta}} \lambda u \pi(u) d u \\
& =\int_{u=x}^{G^{l-1}} \lambda u \pi(u) d u+\int_{u=G^{l-1}}^{\frac{x}{\beta}} \lambda u \pi(u) d u \\
& =\sum_{j=0}^{l-1} \lambda c_{l-j} \frac{b_{j}}{2 a_{j}}\left[e^{a_{j} G^{l-1^{2}}}-e^{a_{j} x^{2}}\right]+\sum_{j=0}^{l-2} \lambda c_{l-1-j} \frac{b_{j}}{2 a_{j}}\left[e^{a_{j} \frac{x^{2}}{\beta^{2}}}-e^{a_{j} G^{l-1^{2}}}\right] \\
\Rightarrow \sum_{j=0}^{l-1} c_{l-j} b_{j} e^{a_{j} x^{2}} & =\sum_{j=0}^{l-1} \lambda c_{l-j} \frac{b_{j}}{2 a_{j}}\left[e^{a_{j} G^{l-1^{2}}}-e^{a_{j} x^{2}}\right]+\sum_{j=0}^{l-2} \lambda c_{l-1-j} \frac{b_{j}}{2 a_{j}}\left[e^{a_{j} \frac{x^{2}}{\beta^{2}}}-e^{a_{j} G^{l-1^{2}}}\right] .
\end{aligned}
$$

This is true in particular for $x=G^{l-1}-\epsilon, \epsilon>0$. Hence we get

$$
\begin{aligned}
\sum_{j=0}^{l-1} c_{l-j} b_{j} e^{a_{j}\left(G^{l-1}-\epsilon\right)^{2}} & =\sum_{j=0}^{l-1} \lambda c_{l-j} \frac{b_{j}}{2 a_{j}}\left[e^{a_{j} G^{l-1^{2}}}-e^{a_{j}\left(G^{l-1}-\epsilon\right)^{2}}\right]+\sum_{j=0}^{l-2} \lambda c_{l-1-j} \frac{b_{j}}{2 a_{j}}\left[e^{a_{j} \frac{(G l-1-\epsilon)^{2}}{\beta^{2}}}-e^{a_{j} G^{l-1^{2}}}\right] \\
c_{l} e^{-\frac{\lambda}{2} G^{l-1^{2}}} & =-\sum_{j=1}^{l-1} c_{l-j} b_{j} e^{a_{j}\left(G^{l-1}\right)^{2}}+\sum_{j=0}^{l-2} \lambda c_{l-1-j} \frac{b_{j}}{2 a_{j}}\left[e^{a_{j} \frac{\left(G^{l-1}\right)^{2}}{\beta^{2}}}-e^{a_{j} G^{l-1^{2}}}\right]
\end{aligned}
$$

where the last relation follows by taking $\epsilon \rightarrow 0$. Thus we obtain $c_{l}$ in terms of $c_{l-j}, j \geq 1$, by noting that by defining $q_{l, j}=-b_{l-j} e^{a_{l-j}\left(G^{l-1}\right)^{2}}+\lambda \frac{b_{l-j}}{2 a_{l-j}}\left[e^{a_{l-j} \frac{\left(G^{l-1}\right)^{2}}{\beta^{2}}}-e^{a_{l-j} G^{l-1^{2}}}\right]$, we can write

$$
\begin{aligned}
c_{l} & =e^{\frac{\lambda}{2} G^{l-1^{2}}} \sum_{j=1}^{l-1} c_{j} q_{l, j} \\
& =e^{\frac{\lambda}{2} G^{l-12}} c_{1} \sum_{j_{1}=1}^{l-1} \sum_{j_{2}=1}^{j_{1}-1} \ldots \sum_{j_{l-2}=1}^{j_{l-3}-1} q_{l, j_{1}} q_{j_{1}, j_{2}} \ldots q_{j_{l-2}, 1} \\
& \triangleq c_{1} Q_{l} .
\end{aligned}
$$

We then get the numerical values of these constants by normalizing the total probability measure to unity. The way this normalization is done is as follows. Let $\Pi_{l}$ be the probability 
mass of the $l^{\text {th }}$ interval, It is seen that

$$
\begin{aligned}
\Pi_{l} & =\int_{u=G^{l}}^{G^{l-1}} \pi(u) d u \\
& =\sum_{j=0}^{l-1} c_{l-j} b_{j} \int_{u=G^{l}}^{G^{l-1}} e^{a_{j} u^{2}} d u \\
& =c_{1} \sum_{j=0}^{l-1} Q_{l-j} b_{j} J_{j}(l)
\end{aligned}
$$

where $J_{j}(l)=\int_{u=G^{l}}^{G^{l-1}} e^{a_{j} u^{2}} d u$ is expressible in terms of upper incomplete Gamma functions. Since $a_{j}<0, \forall j, \int_{u=G^{l}}^{G^{l-1}} e^{a_{j} u^{2}} d u=\int_{u=\left|a_{j}\right|\left(G^{l}\right)^{2}}^{|a|\left(G^{l-1}\right)^{2}} \frac{e^{-u}}{2 \sqrt{\left|a_{j}\right|} \sqrt{u}} d u=\frac{\Gamma\left(0.5,\left|a_{j}\right|\left(G^{l}\right)^{2}\right)-\Gamma\left(0.5,\left|a_{j}\right|\left(G^{l-1}\right)^{2}\right)}{2 \sqrt{\left|a_{j}\right|}}$. Thus,

$$
\begin{gathered}
1=\sum_{l} \Pi_{l}=c_{1} \sum_{l=1}^{m} \sum_{j=0}^{l-1} Q_{l-j} b_{j} J_{j}(l) \\
\Rightarrow c_{l}=Q_{l}\left[\sum_{l=1}^{m} \sum_{j=0}^{l-1} Q_{l-j} b_{j} J_{j}(l)\right]^{-1} .
\end{gathered}
$$

\section{G Proof of Proposition 5}

For $x \in I_{m-k}, k \geq 2$, We get the following Kolmogorov equation

$$
\pi(x) x=\left\{\begin{array}{lc}
\int_{u=x}^{\frac{x}{\beta}} \pi(u) \lambda u d u & x \in I_{m-k}, k \geq 2, \\
\int_{u=x}^{\bar{M}} \pi(u) \lambda u d u+P_{M} \lambda M & x \in I_{m-1} .
\end{array}\right.
$$

Let $E[X]=\int_{x=1}^{M} \pi(x) x d x+P_{M} M$. Dividing both sides of the above Kolmogorov equation by $E[X]$ and defining $\tilde{p} i(x)=\frac{x \pi(x)}{E[X]}$ and $\tilde{P}_{M}=\frac{P_{M} M}{E[X]}$, we get

$$
\tilde{\pi}(x)=\left\{\begin{array}{lc}
\int_{u=x}^{\frac{x}{\beta}} \tilde{\pi}(u) \lambda d u & x \in I_{m-k}, k \geq 2, \\
\int_{u=x}^{\bar{M} x} \tilde{\pi}(u) \lambda d u+\tilde{P}_{M} \lambda & x \in I_{m-1} .
\end{array}\right.
$$

This is the Kolmogorov equation for AIMD protocol under constant loss rate analysed in [5]. The difference is that here the slope of linear increase is unity instead of the parameter $\alpha$ in [5]. We know from [5] that the solution to above Kolmogorov equations is (the complementary distribution function)

$$
\tilde{\pi}(x)=\tilde{P}_{M} \sum_{i=1}^{k} \frac{c_{i}^{(k)} \lambda}{\beta^{i-1}} e^{-x \frac{\lambda}{\beta^{i}-1}},
$$

INRIA 
where

$$
c_{i+1}^{(k)}=\frac{c_{i}^{(k-1)}}{1-\beta^{-i}}
$$

and

$$
c_{1}^{(k)}=e^{\lambda M \beta^{k-1}}\left[\sum_{i=1}^{k-1} c_{i}^{(k-1)} e^{-\lambda M \beta^{k-i}}-\sum_{i=2}^{k} c_{i}^{(k)} e^{-\lambda M \beta^{k-i}}\right]
$$

and

$$
\tilde{P}_{M}=\left[\sum_{i=1}^{m} c_{i}^{(k)} e^{-\lambda M \beta^{k-i}}\right]^{-1}
$$

Hence, for $x \in I_{m-k}$,

$$
\pi(x)=\tilde{P}_{M} E[X] \sum_{i=1}^{k} \frac{c_{i}^{(k)} \lambda}{\beta^{i-1}} \frac{e^{-x \frac{\lambda}{\beta^{i-1}}}}{x}
$$

and

$$
\begin{aligned}
\Pi^{c}(x) & =\int_{u=x}^{M-} \pi(u) d u+P_{M} \\
& =\int_{u=x}^{M \beta^{k-1}} \pi(u) d u+\Pi^{c}\left(M \beta^{k-1}\right) \\
& =\tilde{P}_{M} E[X] \sum_{i=1}^{k} \frac{c_{i}^{(k)} \lambda}{\beta^{i-1}} \int_{u=x}^{M \beta^{k-1}} \frac{e^{-x \frac{\lambda}{\beta^{i-1}}}}{x} d x+\Pi^{c}\left(M \beta^{k-1}\right) \\
& =\tilde{P}_{M} E[X] \sum_{i=1}^{k} \frac{c_{i}^{(k)} \lambda}{\beta^{i-1}} \Gamma\left(0, \frac{x \lambda}{\beta^{i-1}}, \frac{M \lambda}{\beta^{i-k}}\right)+\Pi^{c}\left(M \beta^{k-1}\right) \\
\Pi^{c}\left(M \beta^{k}\right) & =\tilde{P}_{M} E[X] \sum_{i=1}^{k} \frac{c_{i}^{(k)} \lambda}{\beta^{i-1}} \Gamma\left(\frac{M \lambda}{\beta^{i-1-k}}, \frac{M \lambda}{\beta^{i-k}}\right)+\Pi^{c}\left(M \beta^{k-1}\right) .
\end{aligned}
$$

Where $\Gamma(0, a, b)=\int_{t=a}^{b} \frac{e^{-t}}{t} d t$ is the difference of the upper incomplete Gamma functions, $\Gamma(0, a, b)=\Gamma(0, a)-\Gamma(0, b)$ where $\Gamma(0, a)=\int_{t=a}^{\infty} \frac{e^{-t}}{t} d t$. Now,

$$
\begin{aligned}
\Pi^{c}(M \beta) & =\tilde{P}_{M} E[X] c_{1}^{(1)} \lambda \Gamma(0, M \lambda \beta, M \lambda)+P_{M} \\
& =\tilde{P}_{M} E[X] c_{1}^{(1)} \lambda \Gamma(0, M \lambda \beta, M \lambda)+\tilde{P}_{M} \frac{E[X]}{M} .
\end{aligned}
$$

Thus we find $\Pi^{c}\left(M \beta^{k}\right), k \geq 1$ in terms of $E[X]$ since we know the other quantities in the above expressions. Now, since $\Pi^{c}(1)=\Pi^{c}\left(M \beta^{m}\right)=1$, we get the value of $E[X]$, hence $\pi(\cdot)$ for all values of $x$. Proposition 5 thus follows.

$\mathrm{RR} \mathrm{n}^{\circ} 5262$ 


\section{H Proof of Proposition 6}

Consider equation 11. It is seen that,

$$
\begin{aligned}
\pi_{M}(y) & =P_{M} \lambda(M) B(M, y)+\int_{x=y}^{M-} \lambda(x) B(x, y) d \Pi_{M}(x) \quad \forall M \\
\lim _{M \rightarrow \infty} \pi_{M}(y) & =\lim _{M \rightarrow \infty}\left[P_{M} \lambda(M) B(M, y)+\int_{x=y}^{M-} \lambda(x) B(x, y) d \Pi_{M}(x)\right] .
\end{aligned}
$$

Since $\left\{\Pi_{M}(\cdot)\right\}$ is tight, there exists a subsequence $\left\{M_{k}\right\}$ of natural numbers such that $\Pi_{M_{k}}(\cdot)$ converges weakly to another probability measure $\tilde{\Pi}(\cdot)$, i.e., $\Pi_{M_{k}}(\cdot) \Rightarrow \tilde{\Pi}(\cdot)$ (see [20]). Now, if it turns out that the weak limit over this subsequence satisfies

$$
\tilde{\pi}(y) \geq \int_{x=y}^{\infty} \lambda(x) B(x, y) d \tilde{\Pi}(x),
$$

then this implies that $\tilde{\Pi}(\cdot)$ is subinvariant. Since $\tilde{\Pi}(\cdot)$ is a probability measure, it follows that $\tilde{\Pi}([0, \infty))=1<\infty$. Hence, from Proposition 10.1.2 of [23], it follows that $\tilde{\Pi}(\cdot)$ is an invariant measure. Thus we would can prove the Proposition by proving subinvariance of the limiting measure.

Now we show that Equation 25 indeed holds.

$$
\begin{aligned}
& \lim _{k \rightarrow \infty}\left[P_{M_{k}} \lambda\left(M_{k}\right) B\left(M_{k}, y\right)+\int_{x=y}^{M_{k}-} \lambda(x) B(x, y) d \Pi_{M_{k}}(x)\right] \\
& =\lim _{k \rightarrow \infty}\left[P_{M_{k}} \lambda\left(M_{k}\right) B\left(M_{k}, y\right)+\int_{x=y}^{\infty} \lambda(x) I_{\left\{x<M_{k}\right\}} B(x, y) d \Pi_{M_{k}}(x)\right] \\
& \geq \lim _{k \rightarrow \infty} \int_{x=y}^{\infty} \lambda(x) I_{\left\{x<M_{k}\right\}} B(x, y) d \Pi_{M_{k}}(x) \geq \int_{x=y}^{\infty} \lambda(x) B(x, y) d \tilde{\Pi}(x),
\end{aligned}
$$

where the last inequality follows from Fatou's lemma. This also completes the proof.

\section{Proof of Theorem 8}

Let $\pi(y)$ be the density function and $\Pi(y)$ be the distribution function. Note that for $y \in\left(G^{i}, G^{i-1}\right), H(y) \in\left(G^{i-1}, G^{i-2}\right)$. It follows that the Kolmogorov equations are, for $y \geq 0$ :

$$
\pi(y)=\int_{u=y}^{H(y)} \lambda(u) d \Pi(u)
$$

note that we are not having any upper bound on $y$ here. It is possible that the density function $\pi(\cdot)$ does not exist for the boundary points $G^{i}, i>0$. However, continuity of $\Pi(\cdot)$ 
will ensure that these points do not contribute any mass so that we can equate the integral of $\pi(\cdot)$ to unity.

Consider $M>y>G(M)$. For such $y, H(y)=\infty$. Thus,

$$
\begin{aligned}
\pi(y) & =\int_{u=y}^{\infty} \lambda(u) \pi(u) d u \\
& =\lambda(M)(1-\Pi(M))+\int_{u_{1}=y}^{M} \lambda(u) \pi(u) d u \\
& =\lambda(M)(1-\Pi(M)) \sum_{j=0}^{\infty} \int_{u_{1}=y}^{M} \int_{u_{2}=u_{1}}^{M} \ldots \int_{u_{j}=u_{j-1}}^{M} \lambda\left(u_{1}\right) \lambda\left(u_{2}\right) \ldots \lambda\left(u_{j}\right) d u_{j} \ldots d u_{2} d u_{1} .
\end{aligned}
$$

Note that if $\lambda(\cdot)=\lambda$, i.e., a constant state independent loss rate, then

$$
\begin{aligned}
\pi(y) & =\lambda(1-\Pi(M)) e^{\lambda(M-y)}, M>y>G(M) . \\
& \Rightarrow \pi(y)=\theta_{1} e^{-\lambda y}, M>y>G(M),
\end{aligned}
$$

where $\theta_{1}=\lambda(1-\Pi(M)) e^{\lambda M}$.

Now, knowing $\pi(y), M>y>G^{1}$, we will find a bound on $\pi(y), G^{1}>y>G^{2}$.

$$
\begin{aligned}
\pi(y) & =\int_{u=y}^{H(y)} \lambda \pi(u) d u=\int_{u=y}^{G^{1}} \lambda \pi(u) d u+\int_{u=G^{1}}^{H(y)} \lambda \pi(u) d u \\
& \leq \int_{u=y}^{G^{1}} \lambda \pi(u) d u+\int_{u=G^{1}}^{M} \lambda \pi(u) d u=\int_{u=y}^{G^{1}} \lambda \pi(u) d u+\theta_{1}\left(e^{-\lambda G^{1}}-e^{-\lambda M}\right) \\
& \leq \theta_{1}\left(e^{-\lambda G^{1}}-e^{-\lambda M}\right) e^{\lambda\left(G^{1}-y\right)}=\theta_{2} e^{-\lambda y}
\end{aligned}
$$

where $\theta_{2}=\theta_{1}\left(e^{-\lambda G^{1}}-e^{-\lambda M}\right) e^{\lambda G^{1}}=\theta_{1}\left(1-e^{-\lambda l_{1}}\right)$. It is now similarly shown that for $y \in\left[G^{i}, G^{i-1}\right]$,

$$
\pi(y) \leq \theta_{i} e^{-\lambda y}
$$

where $\theta_{i}=\theta_{i-1}\left(1-e^{-\lambda l_{i}}\right)$, i.e.,

$$
\theta_{i}=\theta_{1} \Pi_{j=1}^{i-1}\left(1-e^{-\lambda l_{j}}\right) .
$$

One can also get that for $y>M$,

$$
\pi(y)=\theta_{1} e^{-\lambda y} .
$$

Now, using the fact that $\int_{y=0}^{\infty} \pi(y)=1$, we see that

$$
\begin{aligned}
1 & \leq \theta_{1} \sum_{n=1}^{m}\left[\Pi_{j=1}^{n-1}\left(1-e^{-\lambda l_{j}}\right)\right] \int_{u=G^{n}}^{G^{n-1}} e^{-\lambda y} d y+\frac{\theta_{1}}{\lambda} e^{-\lambda M} \\
1 & \leq \frac{\theta_{1}}{\lambda}\left[\sum_{n=1}^{m}\left[\Pi_{j=1}^{n}\left(1-e^{-\lambda l_{j}}\right)\right] e^{-\lambda G^{n}}+e^{-\lambda M}\right]
\end{aligned}
$$

$\mathrm{RR} \mathrm{n}^{\circ} 5262$ 


$$
\begin{aligned}
1 & \leq \theta_{1} \sum_{n=1}^{m}\left[\Pi_{j=1}^{n-1}\left(1-e^{-\lambda l_{j}}\right)\right] \int_{u=G^{n}}^{G^{n-1}} e^{-\lambda y} d y+\frac{\theta_{1}}{\lambda} e^{-\lambda M} \\
1 & \leq \frac{\theta_{1}}{\lambda}\left[\sum_{n=1}^{m}\left[\Pi_{j=1}^{n}\left(1-e^{-\lambda l_{j}}\right)\right] e^{-\lambda G^{n}}+e^{-\lambda M}\right] \\
\theta_{1} & \geq \lambda\left[\sum_{n=1}^{m}\left[\Pi_{j=1}^{n}\left(1-e^{-\lambda l_{j}}\right)\right] e^{-\lambda G^{n}}+e^{-\lambda M}\right]^{-1} .
\end{aligned}
$$

Since $\lambda(1-\Pi(M)) e^{\lambda M}=\theta_{1}$, Theorem 8 thus follows by using Theorem 6 .

\section{J Proof of Theorem 10}

The Kolmogorov equation for state dependent mark rate is,

$$
\pi(y)=\int_{u=y}^{H(y)} \lambda(u) \pi(u) d u .
$$

For $M>y>G^{1}$, it follows that,

$$
\begin{aligned}
\pi(y) & =\int_{u=y}^{\infty} \lambda(u) \pi(u) d u=\int_{u=y}^{M} \lambda(u) \pi(u) d u+\int_{u=M}^{\infty} \lambda(M) \pi(u) d u \\
& =\int_{u=y}^{M} \lambda(u) \pi(u) d u+\lambda(M)(1-\Pi(u)) \leq \int_{u=y}^{M} \lambda_{1} \pi(u) d u+\theta_{1}^{\prime},
\end{aligned}
$$

where $\lambda_{1}=\sup _{M \geq y \geq G^{1}} \lambda(y)$ and $\theta_{1}^{\prime}=\lambda(M)(1-\Pi(M))$. Thus,

$$
\pi(y) \leq \theta_{1}^{\prime} e^{\lambda_{1}(M-y)}=\theta_{1} e^{-\lambda_{1} y},
$$

with $\theta_{1}=\theta_{1}^{\prime} e^{\lambda_{1} M}$.

Now, for values of $y$ such that $G^{1}>y>G^{2}$,

$$
\begin{aligned}
\pi(y) & =\int_{u=y}^{H(y)} \lambda(u) \pi(u) d u=\int_{u=y}^{G^{1}} \lambda(u) \pi(u) d u+\int_{u=G^{1}}^{H(y)} \lambda(u) \pi(u) d u \\
& \leq \lambda_{2} \int_{u=y}^{G^{1}} \pi(u) d u+\int_{u=G^{1}}^{M} \lambda(u) \theta_{1} e^{-\lambda_{1} u} d u=\lambda_{2} \int_{u=y}^{G^{1}} \pi(u) d u+\theta_{2}^{\prime},
\end{aligned}
$$

where $\lambda_{2}=\sup _{G^{1} \geq y \geq G^{2}} \lambda(y)$ and $\theta_{2}^{\prime}=\int_{u=G^{1}}^{M} \lambda(u) \theta_{1} e^{-\lambda_{1} u} d u$. Thus,

$$
\pi(y) \leq \theta_{2}^{\prime} e^{\lambda_{2}\left(G^{1}-y\right)}=\theta_{2} e^{-\lambda_{2} y}
$$

INRIA 
with $\theta_{2}=\theta_{2}^{\prime} e^{\lambda_{2} G^{1}}$. Proceeding this way, define

$$
\begin{aligned}
\lambda_{i} & =\sup _{G^{i-1} \geq y \geq G^{i}} \lambda(y) \\
\theta_{i}^{\prime} & =\int_{u=G^{i-1}}^{G^{i-2}} \lambda(u) \theta_{i-1} e^{-\lambda_{i-1} u} d u \\
\theta_{i} & =\theta_{i}^{\prime} e^{\lambda_{i} G^{i-1}}
\end{aligned}
$$

giving, in terms of above definition, and with $G^{0} \triangleq M$, for $y \in\left[G^{i}, G^{i-1}\right], 2 \leq i \leq m$,

$$
\pi(y) \leq \theta_{i} e^{-\lambda_{i} y}
$$

and for $y \in\left[G^{1}, M\right]$,

$$
\pi(y) \leq \theta_{1} e^{-\lambda_{1} y}
$$

For $y>M$.

$$
\begin{aligned}
\pi(y) & =\int_{u=y}^{\infty} \lambda(M) \pi(u) d u \\
\Pi^{\prime}(y) & =\lambda(M)[1-\Pi(y)] \\
\frac{d}{d y} \Pi(y) e^{\lambda(M) y} & =\lambda(M) e^{\lambda(M) y} \\
\Pi(y) e^{\lambda(M) y} & =\int_{u=M}^{y} \lambda(M) e^{\lambda(M) u} d u+K
\end{aligned}
$$

where $K=\Pi(M) e^{\lambda(M) M}$. Thus

$$
\begin{aligned}
\Pi(y) & =e^{-\lambda(M) y} \int_{u=M}^{y} \lambda(M) e^{\lambda(M) u} d u+K e^{-\lambda(M) y} \\
\pi(y) & =\theta_{1} e^{-\lambda(M) y} .
\end{aligned}
$$

Define $\kappa_{j}=\frac{\theta_{j}}{1-\Pi(M)}$; note that $\kappa_{j}$ are independent of $1-\Pi(M)$ for a fixed $M$ since $\theta_{j}$ are linear in $1-\Pi(M)$. Now, $\int_{y=0}^{\infty} \pi(y) d y=1$ implies,

$$
\begin{aligned}
1 & \leq \int_{y=M}^{\infty} \pi(y) d y+\sum_{j=1}^{K} \int_{y=G^{j}}^{G^{j-1}} \pi(y) d y \\
1 & \leq\left[\frac{\theta_{1}}{\lambda(M)} e^{-\lambda(M) M}+\sum_{j=1}^{K} \frac{\theta_{j}}{\lambda_{j}}\left(e^{-\lambda_{j} G^{j}}-e^{-\lambda_{j} G^{j-1}}\right)\right] \\
(1-\Pi(M)) & \geq\left[\frac{\kappa_{1}}{\lambda(M)} e^{-\lambda(M) M}+\sum_{j=1}^{K} \frac{\kappa_{j}}{\lambda_{j}}\left(e^{-\lambda_{j} G^{j}}-e^{-\lambda_{j} G^{j-1}}\right)\right]^{-1} .
\end{aligned}
$$

Theorem 10 thus follows by using Conjecture 1 .

$\mathrm{RR} \mathrm{n}^{\circ} 5262$ 


\section{References}

[1] F. Baccelli and D. Hong, "A.I.M.D., Fairness and Fractal Scaling of TCP Traffic," in Proceedings of INFOCOM, New York, June 2002.

[2] P. Brown, "Resource sharing of TCP connections with different round trip times," in Proceedings of INFOCOM, 2000.

[3] S. Asmussen, "Applied probability and queues," Springer, 2003.

[4] A. A. Kherani and A. Kumar, "Stochastic Models for Throughput Analysis of Randomly Arriving Elastic Flows in the Internet," in Proceedings of IEEE INFOCOM, New York, June, 2002.

[5] E. Altman, K. Avratchenkov, C. Barakat and R. Nunez Queija, "State-dependent M/G/1 Type Queueing Analysis for Congestion Control in Data Networks," in Proceedings of IEEE INFOCOM, Anchorage, April, 2001.

[6] E. Altman, K. Avrachenkov and A. A. Kherani, "Stability of Active Queue Management Schemes," Under Preparation.

[7] V. Jacobson and M. J. Karels, "Congestion Avoidance and Control," in Proceedings of the SIGCOMM, Stanford, CA, August, 1988.

[8] Tom Kelly, "Scalable TCP: Improving Performance in Highspeed Wide Area Networks," Submitted for publication, December 2002. Available at http://www-lce.eng.cam.ac.uk/ ctk2/scalable/

[9] S. Athuraliya, V. H. Li, S. H. Low, and Q. Yin, "REM: Active Queue Management," IEEE Network Magazine, Vol. 15, No. 3, May/June, 2001.

[10] G. Vinnicombe, "On the stability of networks operating TCP-like congestion control," IFAC'02 .

[11] A. Misra and T. Ott,"Performance Sensitivity and Fairness of ECN-Aware Modified TCP," Journal of Performance Evaluation (PEVA), Volume 53, Issue 3, August 2003, pp. 255-272.

[12] A. Misra and T. Ott,"The Window Distribution of Idealized TCP Congestion Avoidance with Variable Packet Loss," in Proceedings of IEEE INFOCOM, 1999.

[13] A. A. Kherani and A. Kumar, "Closed Loop Analysis of the Bottleneck Buffer under Adaptive Window Controlled Transfer of HTTP-Like Traffic," in Proceedings of IEEE INFOCOM, San Francisco, 2003.

[14] S. Floyd, "HighSpeed TCP for Large Congestion Windows", RFC 3649, Experimental, December 2003. Available at www.icir.org/floyd/hstcp.html 
[15] A. Budhiraja, F. Hernandez-campos, V. G. Kulkarni and F. D. Smith, "Stochastic Differential Equation for TCP Window size: Analysis and Experimental Validation," Probability in the Engineering and Informational Sciences, Vol. 18, 2004.

[16] R. Bekker, S. Borst, O. Boxma and O. Kella, "Queues with Workload-Dependent Arrival and Service Rates", Queueing Systems: Theory and Applications, Vol 46, 2004.

[17] D. Perry, W. Stadje and S. Zacks, "The M/G/1 Queue with Finite Workload Capacity", Queueing Systems, Vol 39, 2001.

[18] D. P. Heyman and W. Whitt: Limits for Queues as the Waiting Room Grows, 1988.

[19] F. Dufour and O.L.V. Costa: Stability of Piecewise Deterministic Markov Processes, SIAM Journal on Control and Optimization, vol 37 (5), 1483-1502, 1999.

[20] P. Billingsley: Convergence of probability measures, Wiley, New York-London-Sydney, 1968.

[21] M. Davis: Piecewise-deterministic markov processes: A general class of non-diffusion stochastic models, J. R. Statist. Soc. B, 46 (1984), pp. 353-388.

[22] M. Davis: Markov Models and Optimization, Chapman and Hall, London, 1993.

[23] S. Meyn and R. Tweedie: Markov Chains and Stochastic Stability, Springer-Verlag, London, 1993.

[24] A. Misra, T. Ott and J. Baras, " Effect of Exponential Averaging on the Variability of a RED Queue", Proceedings of ICC, 2001.

[25] S. Floyd, R. Gummadi and S. Shenker, "Adaptive RED: An Algorithm for Increasing the Robustness of RED's Active Queue Management, Draft, August 2001.

[26] S. Floyd, and V. Jacobson, "Random Early Detection gateways for Congestion Avoidance", IEEE/ACM Transactions on Networking, V.1 N.4, August 1993, p. 397-413.

[27] M. Mathis, J. Mahdavi, S. Floyd and A. Romanow, "TCP Selective Acknowledgement Options". RFC 2018, April 1996.

[28] L. Kleinrock, "Queueing systems, Vol 1 : Theory", J. Wiley and sons , 1975.

[29] A. A. Borovkov, "Ergodicity and stability of stochastic processes", J. Wiley and sons, 1998.

[30] E. Altman, K. E. Avrachenkov and C. Barakat, "A stochastic model of TCP/IP with stationary random losses", ACM SIGCOMM 2000, Stockholm, Sweden, also in Computer Communication Review, v.30, no.4, October 2000, pp.231-242.

[31] D. Barman, G. Smaragdakiz and I. Matta, "The Effect of Router Buffer Size on HighSpeed TCP Performance", In Proceedings of Globecom 2004.

$\mathrm{RR} \mathrm{n}^{\circ} 5262$ 
[32] E. Altman, K. E. Avrachenkov, A. A. Kherani and B. J. Prabhu, "Performance Analysis and Stochastic Stability of Congestion Control Protocols" INRIA research report, available at http: //www-sop. inria. fr/maestro/personnel/Arzad-Alam. Kherani/me. html

[33] NS-2 Network Simulator, available at http://www. isi. edu/nsnam/ns/ 


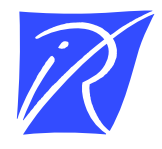

\section{Unité de recherche INRIA Sophia Antipolis 2004, route des Lucioles - BP 93 - 06902 Sophia Antipolis Cedex (France)}

Unité de recherche INRIA Futurs : Parc Club Orsay Université - ZAC des Vignes 4, rue Jacques Monod - 91893 ORSAY Cedex (France)

Unité de recherche INRIA Lorraine : LORIA, Technopôle de Nancy-Brabois - Campus scientifique 615, rue du Jardin Botanique - BP 101 - 54602 Villers-lès-Nancy Cedex (France)

Unité de recherche INRIA Rennes : IRISA, Campus universitaire de Beaulieu - 35042 Rennes Cedex (France)

Unité de recherche INRIA Rhône-Alpes : 655, avenue de l'Europe - 38334 Montbonnot Saint-Ismier (France)

Unité de recherche INRIA Rocquencourt : Domaine de Voluceau - Rocquencourt - BP 105 - 78153 Le Chesnay Cedex (France)

INRIA - Domaine de Voluceau - Rocquencourt, BP 105 - 78153 Le Chesnay Cedex (France)

http://www.inria.fr

ISSN 0249-6399 